\title{
LINEAR HAMILTONIAN BEHAVIORS AND BILINEAR DIFFERENTIAL FORMS*
}

\author{
P. RAPISARDA ${ }^{\dagger}$ AND H. L. TRENTELMAN ${ }^{\ddagger}$
}

\begin{abstract}
We study linear Hamiltonian systems using bilinear and quadratic differential forms. Such a representation-free approach allows us to use the same concepts and techniques to deal with systems isolated from their environment and with systems subject to external influences and allows us to study systems described by higher-order differential equations, thus dispensing with the usual point of view in classical mechanics of considering first- and second-order differential equations only.
\end{abstract}

Key words. linear Hamiltonian systems, two-variable polynomial matrices, bilinear and quadratic differential forms, behavioral system theory

AMS subject classifications. 93A10, 93A30, 93C05, 37J99, 70H50

DOI. $10.1137 / \mathrm{S} 0363012902414664$

1. Introduction. This paper aims to give a unified treatment of linear Hamiltonian systems using the formalism of bilinear and quadratic differential forms introduced in [24]. We consider systems with and without external influences, and we deal with both cases using the same techniques and the same concepts. Moreover, we formulate concepts and study the properties of Hamiltonian systems in a representation-free way, thus dispensing with the usual point of view in mechanics and in physics (see, for example, [1]) of concentrating on first-order representations in the (generalized) coordinates and the (generalized) momenta. Instead of postulating the existence of a function (the Lagrangian, or the Hamiltonian) on the basis of physical considerations (conservation of energy, etc.) and deducing from such a function the equations of motion, we proceed by assuming that a set of linear differential equations with constant coefficients describing the system is given, and we deduce the Hamiltonian nature of the system from such equations, by proving the existence of certain bilinear functionals of the variables of the system and of their derivatives satisfying some additional property. Our approach is of a system-theoretic nature rather than derived from the study of mechanics: as happens in optimal control theory for linear systems, we consider the interplay of (quadratic and bilinear) functionals of the system variables and of the equations of motion as the central object of study when dealing with Hamiltonian systems.

In this paper we also reconcile our point of view with that of classical mechanics by showing how to construct a "generalized Lagrangian" on the basis of the equations of the system, in the sense that the trajectories of the system are stationary with respect to such a quadratic functional of the variables of the system and their derivatives. In this context, the concept of internal force also arises naturally from the equations describing the system: in this paper we show that generalized internal forces can be defined which depend on higher-order derivatives of the external variables and not only first-order ones at most, as happens in classical mechanics.

${ }^{*}$ Received by the editors September 16, 2002; accepted for publication (in revised form) October 17, 2003; published electronically September 18, 2004.

http://www.siam.org/journals/sicon/43-3/41466.html

${ }^{\dagger}$ Department of Mathematics, University of Maastricht, P.O. Box 616, 6200 MD Maastricht, The Netherlands (P.Rapisarda@math.unimaas.nl).

${ }^{\ddagger}$ Research Institute for Mathematics and Computer Science, P.O. Box 800, 9700 AV Groningen, The Netherlands (H.L.Trentelman@math.rug.nl). 
The works more closely connected with the approach proposed in this paper are $[8]$ and $[7,19]$. The approach of $[8]$ is devoted to examining the consequences of the Hamiltonian symmetry of the transfer function on the possibility of realizing a linear system in some special form; the treatment is carried out in the framework of polynomial models. In the present paper we obtain some of the results of [8] when treating Hamiltonian systems with external influences. The work of $[7,19]$ (see also $[6,12,18,20])$ deals with nonlinear systems and consequently has a larger application area than the one illustrated in the present paper. In some cases, most notably in the study of external characterizations of (nonlinear) Hamiltonian systems, such an approach provides results which indeed have a more general nature than some of those presented in this article. However, we believe that the approach presented in this paper, although applicable in its present form only to finite-dimensional linear systems, is relevant for the following reasons. First, the simpler structure of linear systems and the array of algebraic techniques available in the behavioral framework to deal with them allow us to devise constructive methods based on polynomial algebra in order to solve many of the problems arising when considering linear Hamiltonian systems, for example, the computation of "conserved quantities," of special representations, etc. (an example of the technical difficulties involved in solving similar problems in the nonlinear case is given in section III of [6]). Second, the representation-free approach that we pursue allows us to describe systems of a different nature using the same formalism, independent of the domain of application. Such a feature of our approach is especially relevant in view of the potential application of our techniques in the description of possibly infinite-dimensional nonmechanical systems, for example, those arising in the theory of fields. Moreover, proceeding directly from the equations of motion allows us to study Hamiltonianity also for complex systems (for example, those resulting from the interconnection of many simple subsystems), for which the identification of functionals representing the "conserved quantities" is not immediate; this is of particular interest when considering the application of the results presented in this paper to computer-assisted modeling and simulation.

The paper is organized as follows: in section 2 we review some notions regarding linear differential systems and bilinear differential forms, which form the setting in which we study linear Hamiltonian systems. In section 3 we define Hamiltonianity for autonomous systems. In classical mechanics, a Hamiltonian system consists of the trajectories which are stationary with respect to a Lagrangian function; in section 4 we show how such a point of view fits with our definition of Hamiltonianity, and we define the notion of generalized Lagrangian. In section 5 we consider the notion of internal forces, which we propose to see as latent variables arising naturally from the equations describing an autonomous Hamiltonian system. The relationship between internal forces and external variables in an autonomous Hamiltonian system forms the basis for our definition of a controllable Hamiltonian system. In section 6 we discuss our results and outline some directions for future research.

We give a few words on notation. The space of $\mathrm{n}$-dimensional real, respectively, complex, vectors is denoted by $\mathbb{R}^{\mathrm{n}}$, respectively, $\mathbb{C}^{\mathrm{n}}$, and the space of $\mathrm{m} \times \mathrm{n}$ real, respectively, complex, matrices, by $\mathbb{R}^{\mathrm{m} \times \mathrm{n}}$, respectively, $\mathbb{C}^{\mathrm{m} \times \mathrm{n}}$. Whenever one of the two dimensions is not specified, a bullet $\bullet$ is used so that, for example, $\mathbb{C}^{\bullet \times n}$ denotes the set of complex matrices with $\mathrm{n}$ columns and an unspecified number of rows. In order to enhance readability, when dealing with a vector space $\mathbb{R}^{\bullet}$ whose elements are commonly denoted with $w$, we use the notation $\mathbb{R}^{w}$ (note the typewriter font type); similar considerations hold for matrices representing linear operators on such spaces. 
Given two column vectors $x$ and $y$, we denote with $\operatorname{col}(x, y)$ the vector obtained by stacking $x$ over $y$; a similar convention holds for the stacking of matrices with the same number of columns. If $A \in \mathbb{R}^{\mathrm{m} \times \mathrm{n}}$, then $A^{T} \in \mathbb{R}^{\mathrm{n} \times \mathrm{m}}$ denotes its transpose. If $A_{i} \in$ $\mathbb{R}^{\mathrm{k}_{\mathrm{i}} \times \mathrm{k}_{\mathrm{i}}}, i=1, \ldots, \mathrm{m}$, then block $\operatorname{diag}\left(A_{i}\right)_{i=1, \ldots, \mathrm{m}}$ denotes the $\left(\sum_{i=1}^{\mathrm{m}} \mathrm{k}_{\mathrm{i}}\right) \times\left(\sum_{i=1}^{\mathrm{m}} \mathrm{k}_{\mathrm{i}}\right)$ matrix having the $A_{i}$ 's on the main diagonal.

The ring of polynomials with real coefficients in the indeterminate $\xi$ is denoted by $\mathbb{R}[\xi]$; the ring of two-variable polynomials with real coefficients in the indeterminates $\zeta$ and $\eta$ is denoted by $\mathbb{R}[\zeta, \eta]$. A polynomial $p$ in the indeterminate $\xi$ is called even if $p(\xi)=p(-\xi)$ and odd if $p(-\xi)=-p(\xi)$. The space of all $\mathrm{n} \times \mathrm{m}$ polynomial matrices in the indeterminate $\xi$ is denoted by $\mathbb{R}^{\mathrm{n} \times \mathrm{m}}[\xi]$, and that consisting of all $\mathrm{n} \times \mathrm{m}$ polynomial matrices in the indeterminates $\zeta$ and $\eta$ is denoted by $\mathbb{R}^{\mathrm{n} \times \mathrm{m}}[\zeta, \eta]$. Given a matrix $R \in \mathbb{R}^{\mathrm{n} \times \mathrm{m}}[\xi]$, we define $R^{\sim}(\xi):=R^{T}(-\xi) \in \mathbb{R}^{\mathrm{m} \times \mathrm{n}}[\xi]$. If $R(\xi)$ has complex coefficients, then $R^{\sim}(\xi)$ denotes the matrix obtained from $R$ by substituting $-\xi$ in place of $\xi$, transposing, and conjugating.

We denote with $\mathfrak{C}^{\infty}\left(\mathbb{R}, \mathbb{R}^{\mathrm{q}}\right)$ the set of infinitely often differentiable functions from $\mathbb{R}$ to $\mathbb{R}^{\mathrm{q}}$, and we denote with $\mathfrak{D}\left(\mathbb{R}, \mathbb{R}^{\mathrm{q}}\right)$ the subset of $\mathfrak{C}^{\infty}\left(\mathbb{R}, \mathbb{R}^{\mathrm{q}}\right)$ consisting of compact support functions.

Finally, if $K$ is an $\mathrm{n} \times \mathrm{n}$ matrix, the bilinear form on $\mathbb{R}^{\mathrm{n}}$ defined by $\left(x_{1}, x_{2}\right) \mapsto$ $x_{1}^{T} K x_{2}$ is denoted by $\left\langle x_{1}, x_{2}\right\rangle_{K}$. If $K=K^{T}$, then it also induces a quadratic form $x \rightarrow x^{T} K x$, which we denote with $|x|_{K}^{2}$.

2. Basics. In order to make the paper as self-contained as possible, we now illustrate some basic notions regarding linear differential behaviors and bilinear and quadratic differential forms; detailed expositions of such concepts can be found, respectively, in [15] and in [24]. We conclude the section with a brief introduction to Hamiltonian constant matrices, which are relevant in the discussion of state-space representations of Hamiltonian behaviors.

2.1. Linear differential behaviors. A linear differential behavior is a linear subspace $\mathfrak{B}$ of $\mathfrak{C}^{\infty}\left(\mathbb{R}, \mathbb{R}^{\mathrm{w}}\right)$ consisting of all solutions $w$ of a given system of linear constant-coefficient differential equations. Such a set is represented as

$$
R\left(\frac{d}{d t}\right) w=0
$$

where $R \in \mathbb{R}^{\bullet \times \mathrm{w}}[\xi] ;(2.1)$ is called a kernel representation of the behavior $\mathfrak{B}:=\{w \in$ $\mathfrak{C}^{\infty}\left(\mathbb{R}, \mathbb{R}^{\mathrm{w}}\right) \mid w$ satisfies (2.1) $\}$, and $w$ is called the manifest or external variable of $\mathfrak{B}$. The class of all such behaviors is denoted with $\mathfrak{L}^{\mathrm{w}}$.

When modeling physical systems from first principles, we often introduce a number of latent (or auxiliary) variables $\ell$ besides the manifest ones: thus latent variable representations

$$
R\left(\frac{d}{d t}\right) w=M\left(\frac{d}{d t}\right) \ell
$$

are obtained. Equation (2.2) describes the full behavior

$$
\mathfrak{B}_{\mathrm{f}}:=\left\{(w, \ell) \in \mathfrak{C}^{\infty}\left(\mathbb{R}, \mathbb{R}^{\mathfrak{w}+1}\right) \mid(2.2) \text { holds }\right\},
$$

and we call the projection of $\mathfrak{B}_{\mathrm{f}}$ on the $w$ variable, i.e.,

$$
\mathfrak{B}:=\{w \mid \exists \ell \text { such that }(2.2) \text { holds }\},
$$


the manifest behavior associated with (2.2). It can be shown that $\mathfrak{B}$ can also be described in kernel form, i.e., $\mathfrak{B}=\operatorname{ker} R^{\prime}\left(\frac{d}{d t}\right)$ for a suitable $R^{\prime} \in \mathbb{R}^{\bullet \times \mathrm{w}}[\xi]$. The computation of such an $R^{\prime}$ from $R$ and $M$ is called the elimination of the latent variable $\ell$.

When the matrix $R$ in (2.2) is the w-dimensional identity, we call

$$
w=M\left(\frac{d}{d t}\right) \ell
$$

an image representation of $\mathfrak{B}$. A behavior can be represented by (2.3) if and only if each of its kernel representations is associated with a polynomial matrix $R \in \mathbb{R}^{\bullet \times \mathrm{w}}[\xi]$ such that $\operatorname{rank}(R(\lambda))$ is constant for all $\lambda \in \mathbb{C}$, or equivalently, $\mathfrak{B}$ is controllable in the behavioral sense (see Chapter 5 of [15]). The latent variable $\ell$ in (2.3) is called observable from $w$ if $\left[w=M\left(\frac{d}{d t}\right) \ell=0\right] \Longrightarrow[\ell=0]$. It can be shown that this is the case if and only if the matrix $M(\lambda)$ has full column rank for all $\lambda \in \mathbb{C}$.

An important class of behaviors is that of autonomous behaviors, which admit kernel representations (2.1) in which the matrix $R$ is $\mathrm{w} \times \mathrm{w}$ and nonsingular. Given an autonomous behavior $\mathfrak{B} \in \mathcal{L}^{\text {w }}$, the $\mathrm{w} \times$ w matrices associated with any two kernel representations of $\mathfrak{B}$ have the same Smith form (see, for example, section 6.3.3 of [10]). The diagonal elements in such a Smith form are nonzero polynomials called the invariant polynomials of $\mathfrak{B}$; the product of such polynomials of $\mathfrak{B}$ is denoted by $\chi_{\mathfrak{B}}$ and is called the characteristic polynomial of $\mathfrak{B}$.

By permuting the components of $w$ with a permutation matrix $\Pi \in \mathbb{R}^{w \times w}$ if necessary, we can write $\Pi w=\operatorname{col}(u, y)$ with $y$ having $\operatorname{rank}(R)$ components and $u$ having w $-\operatorname{rank}(R)$ components, so that $\mathfrak{B}$ admits the representation $P\left(\frac{d}{d t}\right) y=Q\left(\frac{d}{d t}\right) u$, with $P$ square and nonsingular. We call such a partition of the external variables of $\mathfrak{B}$ an input/output $(i / o)$ partition, $u$ the input variable, $y$ the output variable, and the rational matrix $P^{-1} Q$ the transfer function associated with the given i/o partition. Observe that in general many choices are possible for the permutation matrix $\Pi$ above: the i/o partition is not unique. Observe also that $P^{-1} Q$ is not necessarily a matrix of proper rational functions; however, among all i/o partitions for $\mathfrak{B}$, there exists at least one whose corresponding transfer function is proper.

The number of input variables is an invariant denoted with $\mathrm{m}(\mathfrak{B})$; evidently, the number $\mathrm{p}(\mathfrak{B}):=\mathrm{w}-\mathrm{m}(\mathfrak{B})$ of output variables is also an invariant. If $\mathfrak{B}$ is autonomous, it has no input variables; in other words, $\mathrm{m}(\mathfrak{B})=0$, or equivalently $\mathrm{p}(\mathfrak{B})=$ w. If $\mathfrak{B}$ is controllable, then it admits an observable image representation (2.3) with $M \in \mathbb{R}^{w \times 1}[\xi] ;$ an i/o partition then corresponds to a partition of $M$ as $M=\operatorname{col}(U, Y)$ with $U \in \mathbb{R}^{1 \times 1}[\xi]$ nonsingular; note that $\mathrm{m}(\mathfrak{B})=1$, the dimension of $\ell$. In such a case the transfer function from $u$ to $y$ is the matrix of rational functions $G=Y U^{-1}$.

In this paper we also use the concept of state and of state representation (see [16] for a thorough discussion). A latent variable $\ell$ is a state variable for $\mathfrak{B}$ if and only if $\mathfrak{B}$ admits a representation (2.2) of first order in $\ell$ and zeroth order in $w$ : $E \frac{d \ell}{d t}+F \ell+G w=0$. Such a representation is called a state representation of $\mathfrak{B}$. The minimal number of state variables that can be used in order to represent $\mathfrak{B}$ in state-space form is an invariant called the McMillan degree of $\mathfrak{B}$ and is denoted $\mathrm{n}(\mathfrak{B})$. By combining the notion of state with that of inputs and outputs we arrive at the input/state/output representation ( $i / s / o) \frac{d}{d t} x=A x+B u, y=C x+D u, w=\operatorname{col}(u, y)$.

2.2. Bilinear and quadratic differential forms. In modeling and control problems it is often necessary to study certain functionals of the system variables and their derivatives; when considering linear systems, such functionals are quadratic. 
In [24] the parametrization of such functionals using two-variable polynomial matrices has been studied in detail, resulting in the definition of bilinear and quadratic differential form and in the development of a calculus with applications in stability theory, optimal and $H_{\infty}$-control, and dissipativity theory. Two-variable polynomials and their algebraic properties have been used before in systems theory, for example, by Kalman [11] and Willems and Fuhrmann [22] in the context of stability analysis. We also refer to the pioneering work of Brockett [2] on path independence of integrals of quadratic functionals in the system variables and their derivatives, which prefigures some of the results obtained in [24]. In this section we review those definitions and results of the framework developed in [24] which are used in the rest of this paper.

First, some words about bilinear forms on abstract vector spaces. A bilinear form $\mathcal{L}$ on a vector space $\mathbb{V}$ over $\mathbb{R}$ is a mapping $\mathcal{L}: \mathbb{V} \times \mathbb{V} \rightarrow \mathbb{R}$ that is linear in each of its arguments separately. We sometimes denote a bilinear form as $\left.\mathcal{L}\right|_{\mathbb{V}}$ in order to emphasize its domain. The rank of a bilinear form $\left.\mathcal{L}\right|_{\mathbb{V}}$ equals the number of independent linear functionals $\mathcal{L}(\cdot, v)$, where $v$ ranges over $\mathbb{V}$. A bilinear form $\left.\mathcal{L}\right|_{\mathbb{V}}$ is called nondegenerate if for all $v \in \mathbb{V}$ we have that $\mathcal{L}(\cdot, v)=0$ is equivalent with $v=0$, i.e., $[\mathcal{L}(\mathbb{V}, v)=0] \Leftrightarrow[v=0]$. The bilinear form $\mathcal{L}$ on $\mathbb{V}$ is called skew-symmetric if for all $v_{1}, v_{2} \in \mathbb{V}$ we have $\mathcal{L}\left(v_{1}, v_{2}\right)=-\mathcal{L}\left(v_{2}, v_{1}\right)$. A symplectic space is a pair $(\mathbb{V}, \mathcal{L})$, where $\mathbb{V}$ is a vector space over $\mathbb{R}$ and $\mathcal{L}$ is a nondegenerate, skew-symmetric, bilinear form on $\mathbb{V}$; in such a case $\mathcal{L}$ is called a symplectic form on $\mathbb{V}$. If $\mathbb{V}$ is a finite-dimensional space on $\mathbb{R}$, then nondegenerate symplectic forms are in one-one correspondence with nonsingular skew-symmetric matrices in the sense that for every symplectic form $\mathcal{L}$ there exists a nonsingular $K \in \mathbb{R}^{\mathrm{n} \times \mathrm{n}}$ with $K^{T}=-K$ such that $\mathcal{L}(x, y)=\langle x, y\rangle_{K}$, and conversely, every such $K$ defines a symplectic form on $\mathbb{V}$. Obviously, such $K$ exists only if $\mathrm{n}$ is even.

Next, we examine bilinear differential forms. Let $\Phi \in \mathbb{R}^{\mathrm{w}_{1} \times \mathrm{w}_{2}}[\zeta, \eta]$; then $\Phi(\zeta, \eta)=$ $\sum_{h, k=0}^{N} \Phi_{h, k} \zeta^{h} \eta^{k}$, where $\Phi_{h, k} \in \mathbb{R}^{\mathrm{w}_{1} \times \mathrm{w}_{2}}$ and $N$ is a nonnegative integer. The twovariable polynomial matrix $\Phi$ induces the bilinear functional acting on $w_{1^{-}}$, respectively, $\mathrm{w}_{2}$-dimensional infinitely differentiable trajectories, defined as $L_{\Phi}\left(w_{1}, w_{2}\right)=$ $\sum_{h, k=0}^{N}\left(\frac{d^{h} w_{1}}{d t^{h}}\right)^{T} \Phi_{h, k} \frac{d^{k} w_{2}}{d t^{k}}$. Such a functional is called a bilinear differential form $(\mathrm{BDF}) . L_{\Phi}$ is skew-symmetric, meaning $L_{\Phi}\left(w_{1}, w_{2}\right)=-L_{\Phi}\left(w_{2}, w_{1}\right)$ for all $w_{1}, w_{2}$, if and only if $\Phi$ is a skew-symmetric two-variable polynomial matrix, i.e., if $\mathrm{w}_{1}=\mathrm{w}_{2}$ and $\Phi(\zeta, \eta)=-\Phi^{T}(\eta, \zeta)$.

A two-variable polynomial matrix $\Phi(\zeta, \eta)$ is called symmetric if $\mathrm{w}_{1}=\mathrm{w}_{2}=\mathrm{w}$ and $\Phi(\zeta, \eta)=\Phi^{T}(\eta, \zeta)$. In such a case, $\Phi$ induces also a quadratic functional acting on w-dimensional infinitely smooth trajectories as $Q_{\Phi}(w):=L_{\Phi}(w, w)$. We will call $Q_{\Phi}$ the quadratic differential form (QDF) associated with $\Phi$.

With every $\Phi \in \mathbb{R}^{\mathrm{w}_{1} \times \mathrm{w}_{2}}[\zeta, \eta]$ we associate its coefficient matrix $\tilde{\Phi}$, which is defined as the infinite matrix $\tilde{\Phi}:=\left(\Phi_{i, j}\right)_{i, j=0, \ldots}$. Observe that although $\tilde{\Phi}$ is infinite, only a finite number of its entries are nonzero. Note that $\Phi$ is skew-symmetric if and only if $\tilde{\Phi}^{T}=-\tilde{\Phi}$; also, $\Phi$ is symmetric if and only if $\tilde{\Phi}^{T}=\tilde{\Phi}$.

The association of two-variable polynomial matrices with BDFs and QDFs allows us to develop a calculus that has applications in stability theory, optimal control, and $H_{\infty}$-control. We restrict our attention only to those concepts that are used in this paper. One of them is the map $\partial: \mathbb{R}^{\mathrm{w} \times \mathrm{w}}[\zeta, \eta] \longrightarrow \mathbb{R}^{\mathrm{w} \times \mathrm{w}}[\xi]$ defined by $\partial \Phi(\xi):=$ $\Phi(-\xi, \xi)$. Observe that if $\Phi \in \mathbb{R}^{\mathrm{w} \times \mathrm{w}}[\zeta, \eta]$ is symmetric, then $\partial \Phi$ is para-Hermitian, i.e., $\partial \Phi=(\partial \Phi)^{\sim}$, and if $\Phi$ is skew-symmetric, then $\partial \Phi$ is skew para-Hermitian, i.e., $(\partial \Phi)^{\sim}=-\partial \Phi$. Given a BDF $L_{\Psi}$ we define its derivative as the BDF $L_{\Phi}$ defined by $L_{\Phi}\left(w_{1}, w_{2}\right):=\frac{d}{d t}\left(L_{\Psi}\left(w_{1}, w_{2}\right)\right)$ for all $w_{1}, w_{2}$. In terms of the two-variable polynomial 
matrices associated with the BDFs, the relationship between a BDF and its derivative is expressed as $\Phi(\zeta, \eta)=(\zeta+\eta) \Psi(\zeta, \eta)$. The notion of a derivative of a QDF is analogous and algebraically characterized in the same way; we will not repeat its definition here.

We now discuss the notions of rank and of nondegeneracy of BDFs. Let $\Phi \in$ $\mathbb{R}^{\mathrm{w} \times \mathrm{w}}[\zeta, \eta]$ and $\mathfrak{B} \in \mathfrak{L}^{\mathrm{w}}$. Then the BDF $L_{\Phi}$ induces a bilinear form on the real vector space $\mathfrak{B}$ by assigning to $(v, w) \in \mathfrak{B} \times \mathfrak{B}$ the real number $L_{\Phi}(v, w)(0)$. We denote this bilinear form by $\left.L_{\Phi}\right|_{\mathfrak{B}}$. We can hence speak about the rank and the nondegeneracy of this induced bilinear form. In particular, $\left.L_{\Phi}\right|_{\mathfrak{B}}$ is nondegenerate if for all $w \in \mathfrak{B}$ we have $\left[L_{\Phi}(\mathfrak{B}, w)(0)=0\right] \Leftrightarrow[w=0]$. If $\mathfrak{B}$ is autonomous, then the following result, whose proof is easy and is left to the reader, holds.

Proposition 2.1. Let $\Phi \in \mathbb{R}^{\mathrm{w} \times \mathrm{w}}[\zeta, \eta]$, and let $\mathfrak{B} \in \mathfrak{L}^{\mathrm{w}}$ be autonomous. Let $\frac{d}{d t} x=$ $A x, w=C x$ be a state representation of $\mathfrak{B}$, with full behavior $\mathfrak{B}_{\mathrm{f}}=\left\{(x, w) \mid \frac{d}{d t} x=A x\right.$, $w=C x\}$. Define $N_{\infty}:=\operatorname{col}\left(C A^{i}\right)_{i=0, \ldots} ;$ note that it has an infinite number of rows. Then $L_{\Phi}\left(w_{1}, w_{2}\right)=x_{1}^{T} N_{\infty}^{T} \tilde{\Phi} N_{\infty} x_{2} \forall\left(x_{1}, w_{1}\right),\left(x_{2}, w_{2}\right) \in \mathfrak{B}_{\mathrm{f}}$. Consequently, $\operatorname{rank}\left(\left.L_{\Phi}\right|_{\mathfrak{B}}\right)=\operatorname{rank}\left(N_{\infty}^{T} \tilde{\Phi} N_{\infty}\right)$.

We can now characterize nondegeneracy in terms of rank.

Proposition 2.2. Let $\Phi \in \mathbb{R}^{\mathrm{w} \times \mathrm{w}}[\zeta, \eta]$, and let $\mathfrak{B} \in \mathfrak{L}^{\mathrm{w}}$ be autonomous, with McMillan degree $\mathrm{n}(\mathfrak{B})$. Then $\left.L_{\Phi}\right|_{\mathfrak{B}}$ is nondegenerate if and only if $\operatorname{rank}\left(\left.L_{\Phi}\right|_{\mathfrak{B}}\right) \geq$ $\mathrm{n}(\mathfrak{B})$.

Proof. Let $\frac{d}{d t} x=A x, w=C x$ be a minimal state representation of $\mathfrak{B}$. Then $N_{\infty}^{T} \tilde{\Phi} N_{\infty}$ has size $\mathrm{n}(\mathfrak{B}) \times \mathrm{n}(\mathfrak{B})$. We now prove that $\left.L_{\Phi}\right|_{\mathfrak{B}}$ is nondegenerate if and only if $N_{\infty}^{T} \tilde{\Phi} N_{\infty}$ is nonsingular. This will prove the claim.

(Only if) Assume $N_{\infty}^{T} \tilde{\Phi} N_{\infty} x_{0}=0$. Define $w$ by $w(t)=C e^{A t} x_{0}$. For an arbitrary $w^{\prime}=C e^{A t} x_{0}^{\prime}$, it holds that $L_{\Phi}\left(w^{\prime}, w\right)(0)=x_{0}^{\prime T} N_{\infty}^{T} \tilde{\Phi} N_{\infty} x_{0}=0$; by the nondegeneracy of $L_{\Phi}$, we conclude that $w=0$. Minimality of $\frac{d}{d t} x=A x, w=C x$ implies $x_{0}=0$.

(If) Let $w=C e^{A t} x_{0} \in \mathfrak{B}$, and assume $L_{\Phi}\left(w^{\prime}, w\right)(0)=0$ for all $w^{\prime}=C e^{A t} x_{0}^{\prime} \in \mathfrak{B}$. Then clearly $x_{0}^{\prime T} N_{\infty}^{T} \tilde{\Phi} N_{\infty} x_{0}=0$ for all $x_{0}^{\prime}$, so $N_{\infty}^{T} \tilde{\Phi} N_{\infty} x_{0}=0$. Since $N_{\infty}^{T} \tilde{\Phi} N_{\infty}$ is nonsingular, this implies $x_{0}=0$ and consequently $w=0$.

2.3. Hamiltonian matrices. Given a symplectic form $\langle x, y\rangle_{K}$ on $\mathbb{R}^{\mathrm{n}}$, a linear map $A: \mathbb{R}^{\mathrm{n}} \rightarrow \mathbb{R}^{\mathrm{n}}$ (or matrix $A \in \mathbb{R}^{\mathrm{n} \times \mathrm{n}}$ ) is called Hamiltonian if $\langle A x, y\rangle_{K}+\langle x, A y\rangle_{K}=$ 0 for all $x, y \in \mathbb{R}^{\mathrm{n}}$; equivalently $A^{T} K+K A=0$. For the purposes of this paper, we are especially interested in the invariant polynomials of $\xi I-A$, where $A$ is Hamiltonian; we call them the invariant polynomials of $A$. The relevant result is the following.

Proposition 2.3. Let $A \in \mathbb{R}^{\mathrm{n} \times \mathrm{n}}$ be Hamiltonian. Then its invariant polynomials are either even or odd, and the odd ones can be divided into pairs, so that the multiplicity of zero as a root is the same for the polynomials of each pair.

Proof. In order to prove our statement we use the results of [4]. Such results make use of the concept of elementary divisors of $A$, i.e., the irreducible factors of the invariant polynomials of $\xi I-A$, which are in one-one correspondence with the diagonal blocks appearing in the Jordan form of $A$ (see, for example, section VII.7 of $[9])$.

In Theorem 2.2 of [4] it is proved that if $A$ is Hamiltonian, then its elementary divisors $q_{i} \in \mathbb{C}[\xi]$ are either even polynomials: $q_{i}(\xi)=\left(\xi^{2}+a^{2}\right)^{\mathrm{k}}$, with $a \in \mathbb{R}$; or, if they are not even, then they occur in pairs: as well as $q_{i}(\xi)=(\xi-\lambda)^{\mathrm{k}}, \lambda \in \mathbb{C}$, also $q_{i}^{\sim}(\xi)=(-\xi-\lambda)^{\mathrm{k}}$ appears. Observe that if $\lambda=0$, then such paired elementary divisors are necessarily $\pm \xi^{2 \mathrm{k}+1}$, and if $\lambda \neq 0$, then they are coprime with each other.

Conclude from these remarks that $\xi I-A$ is Smith equivalent to a diagonal form, where on the diagonal appear either even polynomials, or pairs of polynomials of the 
form $\xi^{2 \mathrm{k}+1}$. We now use the argument of Theorem 2 of [5] in order to prove the claim. Let $1 \leq \mathrm{k} \leq \mathrm{n}$, and consider the set $M_{\mathrm{k}}$ consisting of all $\mathrm{k} \times \mathrm{k}$ minors of $\xi I-A$; observe that $M_{\mathrm{k}}$ contains only even or odd polynomials. Now consider the greatest common divisor $\Delta_{\mathrm{k}}$ of the polynomials in $M_{\mathrm{k}}$, and observe that for every $m \in M_{\mathrm{k}}$ there exists $m^{\prime} \in \mathbb{R}[\xi]$ such that $m=\Delta_{\mathrm{k}} m^{\prime}$; moreover, since $m$ is either even or odd, it holds that $m^{\sim}= \pm m=\Delta_{\mathrm{k}}^{\sim} m^{\prime \sim}$. It follows that $\Delta_{\mathrm{k}}^{\sim}$ divides every polynomial in $M_{\mathrm{k}}$. Consequently $\Delta_{\mathrm{k}}^{\sim}$ divides $\Delta_{\mathrm{k}}$, and by symmetry also the converse holds. It follows that $\Delta_{\mathrm{k}}= \pm \Delta_{\mathrm{k}}^{\sim}$. Since the invariant polynomials are obtained dividing $\Delta_{\mathrm{k}}$ by $\Delta_{\mathrm{k}-1}$ (with $\Delta_{0}:=1$ ), it follows that the invariant polynomials of $\xi I-A$ are either even or odd.

In order to prove the claim regarding the paired odd polynomials, assume by contradiction that there exists a pair of odd invariant polynomials of $A$ for which zero is a root with multiplicity $2 \mathrm{k}_{1}+1$ and $2 \mathrm{k}_{2}+1$, respectively, $\mathrm{k}_{1} \neq \mathrm{k}_{2}$, and which cannot be paired otherwise. Observe that $\xi^{2 \mathrm{k}_{1}+1}$ and $\xi^{2 \mathrm{k}_{2}+1}$ are elementary divisors of $A$. Conclude that in the Jordan form of $A$ there are two blocks associated with zero, of dimension $2 \mathrm{k}_{1}+1$ and $2 \mathrm{k}_{2}+1$, respectively, which cannot be paired otherwise. This, however, is in contradiction with the results of [4] on the elementary divisors. This concludes the proof.

3. Autonomous Hamiltonian systems. The definition of an autonomous Hamiltonian system is as follows.

Definition 3.1. Let $\mathfrak{B} \in \mathcal{L}^{\text {w }}$ be autonomous. $\mathfrak{B}$ is called Hamiltonian if there exists a bilinear differential form $L_{\Psi}$, such that

(i) $\frac{d}{d t} L_{\Psi}\left(w_{1}, w_{2}\right)=0$ for all $w_{1}, w_{2} \in \mathfrak{B}$;

(ii) $L_{\Psi}$ is skew-symmetric;

(iii) $\left.L_{\Psi}\right|_{\mathfrak{B}}$ is nondegenerate.

In Definition 3.1 no assumption on the number w of external variables of $\mathfrak{B}$ is made. This point of view is in contrast with the usual definition of an autonomous Hamiltonian system, in which a symplectic structure on the space of the external variables (and consequently, an even number of such variables) is assumed. We believe that in order to investigate linear, finite-dimensional Hamiltonian systems, Definition 3.1 is a natural starting point, more so than the classical one in mechanics, as argued in the following examples.

Example 3.2. Consider a spring-mass system without friction, with behavior $\mathfrak{B}$ represented by the equation $m \frac{d^{2}}{d t^{2}} w$ where $w$ is the displacement from the equilibrium position. The BDF $L_{\Psi}$ induced by $\Psi(\zeta, \eta)=m(\zeta-\eta)$ is skew-symmetric. In fact, $L_{\Psi}\left(w_{1}, w_{2}\right)=m\left(\frac{d}{d t} w_{1}\right) w_{2}-m\left(\frac{d}{d t} w_{2}\right) w_{1}$. It is easily seen that $\frac{d}{d t} L_{\Psi}\left(w_{1}, w_{2}\right)=0$ for all $w_{1}, w_{2} \in \mathfrak{B}$. Also

$$
L_{\Psi}\left(w_{1}, w_{2}\right)(0)=\left(\begin{array}{c}
w_{1}(0) \\
\frac{d}{d t} w_{1}(0)
\end{array}\right)^{T}\left(\begin{array}{cc}
0 & -m \\
m & 0
\end{array}\right)\left(\begin{array}{c}
w_{2}(0) \\
\frac{d}{d t} w_{2}(0)
\end{array}\right)
$$

which clearly defines a nondegenerate bilinear form on $\mathfrak{B}$. It follows that this springmass system with only one external variable is Hamiltonian according to Definition 3.1. It is difficult to understand why, in order to study the Hamiltonianity of such a system from the point of view of classical mechanics, one should first transform the natural second-order differential equation description into a first-order representation in which the position and the momentum of the mass are the external variables and then study the symplectic structure of the resulting state-space system.

The previous example illustrates but one situation in which a representation-free definition of Hamiltonianity appears to be more natural than the classical one. The 
argument for a definition of Hamiltonianity independent of the particular representation at hand becomes even stronger if one realizes that very often a dynamical system is described by a set of higher-order differential equations, obtained, for example, after elimination of auxiliary variables. The following example illustrates this point.

Example 3.3. Consider two masses $m_{1}$ and $m_{2}$ attached to springs with constants $k_{1}$ and $k_{2}$. The first mass is connected to the second one via the first spring, and the second mass is connected to a "wall" with the second spring. Denote by $w_{1}$ and $w_{2}$ the positions of the masses. Then we can write down the equation of the system as $m_{1} \frac{d^{2} w_{1}}{d t^{2}}+k_{1} w_{1}-k_{1} w_{2}=0,-k_{1} w_{1}+m_{2} \frac{d^{2} w_{2}}{d t^{2}}+\left(k_{1}+k_{2}\right) w_{2}=0$. Eliminate $w_{2}$ from the equations and take the position $w_{1}$ of the first mass as our external variable $w$. The behavior $\mathfrak{B}$ of $w$ is represented by

$$
m_{1} m_{2} \frac{d^{4}}{d t^{4}} w+\left(k_{1} m_{1}+k_{2} m_{1}+k_{1} m_{2}\right) \frac{d^{2}}{d t^{2}} w+k_{1} k_{2} w=0 .
$$

In order to simplify the notation, define $r_{0}:=k_{1} k_{2}, r_{2}:=k_{1} m_{1}+k_{2} m_{1}+k_{1} m_{2}$, and $r_{4}:=m_{1} m_{2}$, so that the equation describing $w$ can be rewritten as $r\left(\frac{d}{d t}\right) w=0$, where $r(\xi):=r_{0}+r_{2} \xi^{2}+r_{4} \xi^{4}$. Define the skew-symmetric polynomial $\Psi(\zeta, \eta)$ by $\Psi(\zeta, \eta)=$ $r_{2}(\zeta-\eta)+r_{4}\left(\zeta^{3}-\eta^{3}\right)+r_{4}\left(\zeta \eta^{2}-\zeta^{2} \eta\right)$. Observe that $(\zeta+\eta) \Psi(\zeta, \eta)=r(\zeta)-r(\eta)$, and consequently $\frac{d}{d t} L_{\Psi}(v, w)=\left(r\left(\frac{d}{d t}\right) v\right)^{T} w-v^{T}\left(r\left(\frac{d}{d t}\right) w\right)=0$ for all $v, w \in \mathfrak{B}$. Moreover, $\Psi(\eta, \zeta)=-\Psi(\zeta, \eta)$, implying that the $\operatorname{BDF} L_{\Psi}(v, w)$ is skew-symmetric. Finally, since the coefficient matrix $\tilde{\Psi}$ of $\Psi(\zeta, \eta)$ is nonsingular, $L_{\Psi}(v, w)(0)$ clearly defines a nondegenerate bilinear form on $\mathfrak{B}$. Hence the behavior $\mathfrak{B}$ is a Hamiltonian system in the sense of Definition 3.1.

The following theorem gives conditions under which a given autonomous linear differential behavior is Hamiltonian.

THeOREM 3.4. Let $\mathfrak{B} \in \mathcal{L}^{\text {w }}$ be autonomous. The following conditions are equivalent:

(1) $\mathfrak{B}$ is Hamiltonian;

(2) every invariant polynomial of $\mathfrak{B}$ is either even or odd, and the odd invariant polynomials can be divided into pairs so that the multiplicity of zero as a root is the same for the polynomials of each pair;

(3) there exists a minimal state representation $\frac{d}{d t} x=A x, w=C x$ of $\mathfrak{B}$, and a symplectic form $\langle\cdot, \cdot\rangle_{K}$ on the state space $\mathbb{R}^{\mathrm{n}(\mathfrak{B})}$ such that $A$ is a Hamiltonian matrix;

(4) for any minimal state representation $\frac{d}{d t} x=A x, w=C x$ of $\mathfrak{B}$ there exists a symplectic form $\langle\cdot, \cdot\rangle_{K}$ on the state space $\mathbb{R}^{\mathrm{n}(\mathfrak{B})}$ such that $A$ is a Hamiltonian matrix.

Proof. We prove $(1) \Rightarrow(4) \Rightarrow(3) \Rightarrow(2) \Rightarrow(1)$.

$((1) \Rightarrow(4))$ Let $L_{\Psi}$ satisfy Definition 3.1. Let $\frac{d}{d t} x=A x, w=C x$ be a minimal state representation of $\mathfrak{B}$. From Proposition 2.1 we have that for $K_{\Psi}:=N_{\infty}^{T} \tilde{\Psi} N_{\infty} \in$ $\mathbb{R}^{\mathrm{n}(\mathfrak{B}) \times \mathrm{n}(\mathfrak{B})}$ we have $L_{\Psi}\left(w_{1}, w_{2}\right)=x_{1}^{T} K_{\Psi} x_{2}$ for all $\left(w_{i}, x_{i}\right)(i=1,2)$ satisfying the system equations and, moreover, $\operatorname{rank}\left(\left.L_{\Psi}\right|_{\mathfrak{B}}\right)=\operatorname{rank}\left(K_{\Psi}\right)$. Since $\left.L_{\Psi}\right|_{\mathfrak{B}}$ is nondegenerate, it follows from Proposition 2.2 that $\operatorname{rank}\left(K_{\Psi}\right)=\mathrm{n}(\mathfrak{B})$. Consequently $K_{\Psi}$ is nonsingular. Conclude from $\frac{d}{d t} L_{\Psi}\left(w_{1}, w_{2}\right)=0$ for all $w_{1}, w_{2} \in \mathfrak{B}$ that $x_{1}^{T}\left(A^{T} K_{\Psi}+K_{\Psi} A\right) x_{2}=0$ for all $x_{i}$ such that $\frac{d}{d t} x_{i}=A x_{i}, i=1,2$. This implies $A^{T} K_{\Psi}+K_{\Psi} A=0$.

$((4) \Rightarrow(3))$ is trivial.

$((3) \Rightarrow(2))$ Conclude from Proposition 2.3 that the invariant polynomials of $A$ are either even or odd and that the odd ones come in pairs and have zero as a root with the same multiplicity. Now let $R \in \mathbb{R}^{\bullet \times w}[\xi]$ be such that $\mathfrak{B}=\operatorname{ker} R\left(\frac{d}{d t}\right)$. Since $\mathfrak{B}$ is 
autonomous, $R$ has full column rank. Let $\lambda_{i}, i=1, \ldots$, w, be the invariant polynomials of $R$. Let $U$ and $V$ be the unimodular matrices such that $R=U \operatorname{col}(\Lambda, 0) V$, with $\Lambda:=\operatorname{diag}\left(\lambda_{i}\right)_{i=1, \ldots, \mathrm{w}}$, the Smith form of $R$. Then $\mathfrak{B}$ is alternatively represented by $w=V\left(\frac{d}{d t}\right)^{-1} w^{\prime}, 0=\Lambda\left(\frac{d}{d t}\right) w^{\prime}$. We now construct a minimal state representation of $\mathfrak{B}$. For $i=1, \ldots$, w, define $A_{c, i}$ to be the companion matrix associated with the $i$ th invariant polynomial; also, let $C_{i}$ be the first vector of the canonical basis of $\mathbb{R}^{\operatorname{deg}\left(\lambda_{i}\right)}$. Define $\hat{A}:=\operatorname{block} \operatorname{diag}\left(A_{c, i}\right)_{i=1, \ldots, \mathrm{w}}, \hat{C}:=\operatorname{block} \operatorname{diag}\left(C_{i}\right)_{i=1, \ldots, \mathrm{w}}$. Then $\frac{d}{d t} x=\hat{A} x$, $w^{\prime}=\hat{C} x$ is a minimal state representation of $\operatorname{ker} \Lambda\left(\frac{d}{d t}\right)$. In order to come up with a minimal state representation of $\mathfrak{B}$, let $V^{-1}(\xi)=V_{0}+V_{1} \xi+\cdots+V_{N} \xi^{N}$; then it is easy to verify that $\frac{d}{d t} x=\hat{A} x, w=\left(V_{0} \hat{C}+V_{1} \hat{C} \hat{A}+\cdots+V_{N} \hat{C} \hat{A}^{N-1}\right) x$ is such a representation. Observe also that $\xi I-\hat{A}$ has the same invariant polynomials of $\xi I-A$, since $\frac{d}{d t} x=A x, w=C x$ is another minimal state-space representation of $\mathfrak{B}$ (see Lemma 6.3-19 of [10]). This concludes the proof of $((3) \Rightarrow(2)$ ).

$((2) \Rightarrow(1))$ We first derive a special representation of $\mathfrak{B}$. Let $\mathfrak{B}=\operatorname{ker} R\left(\frac{d}{d t}\right)$ be a minimal kernel representation of $\mathfrak{B}$, and let $R=U \Delta V$ be a Smith decomposition of $R$, with $\Delta$ the diagonal matrix of the invariant polynomials. Denote the even invariant polynomials of $\mathfrak{B}$ with $\lambda_{i}$, where $i=1, \ldots$, e. We denote the odd, paired, invariant polynomials with $\mu_{i}, i=1, \ldots, \mathrm{w}-\mathrm{e}$ (observe that $\mathrm{w}-\mathrm{e}$ is even). Now reorder if necessary the invariant polynomials so that the first e diagonal entries of $\Delta$ are the $\lambda_{i}$ and the last $\mathrm{w}-\mathrm{e}$ the $\mu_{i}$. From the division property of the invariant polynomials and from the pairing property of the odd invariant polynomials, it follows that we can write $\mu_{2 i+1}(\xi)=\xi \pi_{i}(\xi), \mu_{2 i+2}(\xi)=\xi \pi_{i}(\xi) g_{i}(\xi) g_{i}(-\xi)$ with $\pi_{i}$ even and $g_{i}$ and $g_{i}^{\sim}$ coprime; in other words, $g(0) \neq 0$. (Observe that $g_{i}$ in general has complex coefficients, and consequently in the following it may be necessary to work with polynomial matrices with complex coefficients.)

From these considerations it follows that each $2 \times 2$ submatrix $\operatorname{diag}\left(\mu_{2 i+1}, \mu_{2 i+2}\right)$, $i=0, \ldots, \frac{\mathrm{w}-\mathrm{e}}{2}-1$, is Smith-equivalent to

$$
\Delta_{i}^{\prime}(\xi)=\left(\begin{array}{cc}
0 & -\xi \pi_{i}(\xi) g_{i}(-\xi) \\
\xi \pi_{i}(\xi) g_{i}(\xi) & 0
\end{array}\right)
$$

in other words, there exist unimodular matrices $T_{i}$ and $S_{i} \in \mathbb{C}^{2 \times 2}[\xi]$ such that $\Delta_{i}^{\prime}=$ $T_{i} \operatorname{diag}\left(\mu_{2 i+1}, \mu_{2 i+2}\right) S_{i}$. Now define

$$
T=\operatorname{diag}\left(I_{r}, T_{1}, \ldots, T_{\frac{\mathrm{w}-\mathrm{e}}{2}}\right)
$$

and

$$
S=\operatorname{diag}\left(I_{r}, S_{1}, \ldots, S_{\frac{\mathrm{w}-\mathrm{e}}{2}}\right),
$$

and observe that $T \Delta S=\operatorname{diag}\left(\Lambda, \Delta_{1}^{\prime}, \ldots, \Delta_{\frac{\mathrm{v}-\mathrm{e}}{2}}^{\prime}\right)=: \hat{\Delta}$, where $\Lambda:=\operatorname{diag}\left(\lambda_{1}, \ldots, \lambda_{\mathrm{e}}\right)$. Conclude that $\left(S^{-1} V\right)^{\sim} T U^{-1} R=\left(S^{-1} V\right)^{\sim} \hat{\Delta} S^{-1} V=: R^{\prime} \in \mathbb{R}^{\mathrm{w} \times \mathrm{w}}[\xi]$ is another kernel representation of $\mathfrak{B}$ satisfying $R^{\prime}=R^{\prime \sim}$. It is such a representation that we use in order to come up with a BDF as in Definition 3.1.

Consider the unimodular transformation of the external variables represented by $w^{\prime}:=\left(S^{-1} V\right)\left(\frac{d}{d t}\right) w$, and observe that the $i$ th component of $w^{\prime}, i=1, \ldots, \mathrm{e}$, satisfies $\lambda_{i}\left(\frac{d}{d t}\right) w_{i}^{\prime}=0$, while the remaining components satisfy $\Delta_{i}^{\prime}\left(\frac{d}{d t}\right) \operatorname{col}\left(w_{i}^{\prime}, w_{i+1}^{\prime}\right)=0, i=$ $0, \ldots, \frac{\mathrm{w}-\mathrm{e}}{2}-1$. We proceed to construct a skew-symmetric BDF $L_{\Psi_{i}}$ acting on the $i$ th component $w_{i}^{\prime}$ and satisfying Definition 3.1. From such BDFs we will construct a $\mathrm{BDF}$ for $\mathfrak{B}$ with the right properties. 
We begin by computing such a BDF for the case of even invariant polynomials. Since $\lambda_{i}-\lambda_{i}^{\sim}=0$, it follows from Theorem 3.1 of [24] that there exists $\Psi_{i} \in \mathbb{R}[\zeta, \eta]$ such that $(\zeta+\eta) \Psi_{i}(\zeta, \eta)=\lambda_{i}(\zeta)-\lambda_{i}(\eta)$. Observe that $\Psi_{i}(\zeta, \eta)$ is skew-symmetric and moreover, $\frac{d}{d t} L_{\Psi_{i}}\left(w_{1}, w_{2}\right)=0$ for all $w_{1}, w_{2} \in \operatorname{ker} \lambda_{i}\left(\frac{d}{d t}\right)$. We now prove that $L_{\Psi_{i}}$ is nondegenerate. Write $\lambda_{i}(\xi)=\lambda_{i 0}+\lambda_{i 2} \xi^{2}+\cdots+\lambda_{i, 2 n_{i}} \xi^{2 n_{i}}$. It is easy to verify that the coefficient matrix $\tilde{\Psi}_{i}$ of $\Psi(\zeta, \eta)$ is a $2 n_{i} \times 2 n_{i}$ left-upper-triangular matrix with $\pm \lambda_{i, 2 n_{i}}$ on the main antidiagonal; as a consequence, the bilinear differential form $\left.L_{\Psi_{i}}\right|_{\operatorname{ker}\left(\lambda_{i}\left(\frac{d}{d t}\right)\right)}$ is nondegenerate. This settles the case of even invariant polynomials.

We now examine the case of paired odd invariant polynomials. Observe first that since $\pi_{i}$ is even, the two-variable polynomial $\zeta \pi_{i}(\zeta) g_{i}(\zeta)+\eta \pi_{i}(\eta) g_{i}(-\eta)$ is divisible by $\zeta+\eta$. Now define the skew-symmetric two-variable polynomial matrix $\Psi_{i}(\zeta, \eta) \in$ $\mathbb{C}^{2 \times 2}[\zeta, \eta]$ as

$$
\Psi_{i}(\zeta, \eta):=\left(\begin{array}{cc}
0 & \frac{\zeta \pi_{i}(\zeta) g_{i}(\zeta)+\eta \pi_{i}(\eta) g_{i}(-\eta)}{\zeta+\eta} \\
-\frac{\zeta \pi_{i}(\zeta) g_{i}(-\zeta)+\eta \pi_{i}(\eta) g_{i}(\eta)}{\zeta+\eta} & 0
\end{array}\right) .
$$

We observe that $\frac{d}{d t} L_{\Psi_{i}}\left(\operatorname{col}\left(w_{i}^{\prime}, w_{i+1}^{\prime}\right), \operatorname{col}\left(\bar{w}_{i}^{\prime}, \bar{w}_{i+1}^{\prime}\right)\right)=0$ for each pair $\operatorname{col}\left(w_{i}^{\prime}, w_{i+1}^{\prime}\right)$, $\operatorname{col}\left(\bar{w}_{i}^{\prime}, \bar{w}_{i+1}^{\prime}\right)$ of trajectories in ker $\Delta_{i}^{\prime}\left(\frac{d}{d t}\right)$. We now prove that $\left.L_{\Psi_{i}}\right|_{\operatorname{ker} \Delta_{i}^{\prime}\left(\frac{d}{d t}\right)}$ is nondegenerate.

Let $\operatorname{deg}\left(\pi_{i}\right)=2 K_{i}, \operatorname{deg}\left(g_{i}\right)=L_{i}$. It is a matter of straightforward verification to prove that the coefficients of the terms $\zeta^{k} \eta^{L_{i}+2 K_{i}-k}, k=0, \ldots, L_{i}+2 K_{i}$, in $\frac{\zeta \pi_{i}(\zeta) g_{i}(\zeta)+\eta \pi_{i}(\eta) g_{i}(-\eta)}{\zeta+\eta}$ are equal to $g_{i, L_{i}} \pi_{i, 2 K_{i}} \neq 0$. It follows that the coefficient matrix $\tilde{\Psi}_{i}$ is a block-left-upper-triangular matrix with on the diagonal $2 \times 2$ blocks of the form

$$
(-1)^{k}\left(\begin{array}{cc}
0 & g_{i, L_{i}} \pi_{i, 2 K_{i}} \\
-g_{i, L_{i}} \pi_{i, 2 K_{i}} &
\end{array}\right)
$$

We conclude from this that $\tilde{\Psi}_{i}$ is nonsingular, and consequently the bilinear form $\left.L_{\Psi_{i}}\right|_{\text {ker } \Delta_{i}^{\prime}\left(\frac{d}{d t}\right)}$ is nondegenerate. This settles the case of paired odd invariant polynomials.

In order to complete the proof of the claim $(2) \Rightarrow(1)$, assume $\Psi_{i}(\zeta, \eta)$ has been constructed as described above; now define

$$
\Psi(\zeta, \eta):=\left(S^{-1} V\right)(\zeta)^{T} \operatorname{diag}\left(\Psi_{i}(\zeta, \eta)\right)\left(S^{-1} V\right)(\eta) \in \mathbb{R}^{\mathrm{q} \times \mathrm{q}}[\zeta, \eta],
$$

where $S, V$ are the unimodular matrix involved in obtaining the special decomposition of $R^{\prime}$. (Observe that if some $\Psi_{i}(\zeta, \eta)$ has complex coefficients, then transposition and complex conjugation are required.) $\Psi(\zeta, \eta)$ induces a skew-symmetric $\operatorname{BDF} L_{\Psi}$ whose derivative is zero along $\mathfrak{B}$. The nondegeneracy of $\left.L_{\Psi}\right|_{\mathfrak{B}}$ follows immediately from the nondegeneracy of the forms $\left.L_{\Psi_{i}}\right|_{\operatorname{ker} \lambda_{i}\left(\frac{d}{d t}\right)}$ and $\left.L_{\Psi_{i}}\right|_{\operatorname{ker} \Delta_{i}^{\prime}\left(\frac{d}{d t}\right)}$. This concludes the proof.

4. Hamiltonian systems and the Euler-Lagrange equations. In section 3 we introduced the notion of Hamiltonianity on the basis of the interplay of a skewsymmetric BDF with the dynamics of the system, without reference to the notion of Lagrangian as occurs in classical mechanics. In this section we reconcile the classical point of view with our standpoint.

We begin by introducing the notion of stationarity of a trajectory with respect to a QDF. Let $\Phi \in \mathbb{R}^{\mathrm{w} \times \mathrm{w}}[\zeta, \eta]$ be symmetric and consider the corresponding QDF 
$Q_{\Phi}(w)$ on $\mathfrak{C}^{\infty}\left(\mathbb{R}, \mathbb{R}^{w}\right)$. For a given $w$ we define the cost degradation of adding the compact-support function $\delta \in \mathfrak{D}\left(\mathbb{R}, \mathbb{R}^{w}\right)$ to $w$ as

$$
J_{w}(\delta):=\int_{-\infty}^{+\infty}\left(Q_{\Phi}(w+\delta)-Q_{\Phi}(w)\right) d t
$$

The cost degradation equals $J_{w}(\delta)=\int_{-\infty}^{+\infty} Q_{\Phi}(\delta) d t+2 \int_{-\infty}^{+\infty} L_{\Phi}(w, \delta) d t$, and we call the second integral on the right of the equality sign the variation associated with $w$. It defines a linear functional which associates with every $\delta \in \mathfrak{D}\left(\mathbb{R}, \mathbb{R}^{\mathrm{w}}\right)$ a real number $2 \int_{-\infty}^{+\infty} L_{\Phi}(w, \delta) d t$. We call $w$ a stationary trajectory of $Q_{\Phi}$ if the variation associated with $w$ is the zero functional. The following proposition establishes a representation of all stationary trajectories of given QDF $Q_{\Phi}$. Recall that, for a given two-variable polynomial matrix $\Phi(\zeta, \eta), \partial \Phi(\xi)$ is defined as the one-variable polynomial matrix $\Phi(-\xi, \xi)$.

Proposition 4.1. Let $\Phi(\zeta, \eta) \in \mathbb{R}^{\mathrm{w} \times \mathrm{w}}[\zeta, \eta]$ be symmetric. Then $w \in \mathfrak{C}^{\infty}\left(\mathbb{R}, \mathbb{R}^{\mathrm{w}}\right)$ is a stationary trajectory of the $Q D F Q_{\Phi}$ if and only if $w$ satisfies the differential equation

$$
\partial \Phi\left(\frac{d}{d t}\right) w=0
$$

Proof. Factor $\Phi(\zeta, \eta)=M^{T}(\zeta) \Sigma M(\eta)$, with $\Sigma$ a nonsingular signature matrix, and $M(\xi)=M_{0}+M_{1} \xi+M_{2} \xi^{2}+\cdots+M_{L} \xi^{L}$ (see section 3 of [24]). Integrating by parts on $\delta \in \mathfrak{D}\left(\mathbb{R}, \mathbb{R}^{\mathrm{w}}\right)$, the variation $\int_{-\infty}^{+\infty}\left(M\left(\frac{d}{d t}\right) w\right)^{T} \Sigma M\left(\frac{d}{d t}\right) \delta d t$ is seen to be equal to

$$
\begin{gathered}
\left.\sum_{k=1}^{L} \sum_{j=k}^{L}(-1)^{k-1} \delta^{(j-k)} M_{j}^{T} \Sigma\left(M\left(\frac{d}{d t}\right) w\right)^{(k-1)}\right|_{-\infty} ^{+\infty} \\
+\int_{-\infty}^{+\infty} \delta^{T}\left(M\left(-\frac{d}{d t}\right)^{T} \Sigma M\left(\frac{d}{d t}\right) w\right) d t .
\end{gathered}
$$

Such a quantity is zero if and only if $M\left(-\frac{d}{d t}\right)^{T} \Sigma M\left(\frac{d}{d t}\right) w=0$; equivalently, $\partial \Phi\left(\frac{d}{d t}\right) w=0$.

From the classical theory of calculus of variations it is well known that the stationary trajectories for a given functional can be characterized in terms of the so-called higher-order Euler equations, often called the Euler-Poisson equations. If the functional is given by a $\mathrm{QDF} Q_{\Phi}$, then (4.1) can indeed be interpreted as a classical Euler-Poisson equation. In order to verify this, let $\tilde{\Phi}_{\text {eff }}:=\left(\Phi_{k, \ell}\right)_{k, \ell=0, \ldots, L}$. Now observe that $Q_{\Phi}(w)$ can be written as $F\left(w, w^{(1)}, w^{(2)}, \ldots, w^{(L)}\right)$, with the functional $F: \mathbb{R}^{\mathrm{w}} \times \mathbb{R}^{\mathrm{w}} \times \cdots \times \mathbb{R}^{\mathrm{w}} \rightarrow \mathbb{R}$ defined by

$$
F\left(w_{0}, w_{1}, w_{2}, \ldots, w_{L}\right):=\operatorname{col}\left(w_{0}, w_{1}, w_{2}, \ldots, w_{L}\right)^{T} \tilde{\Phi}_{\text {eff }} \operatorname{col}\left(w_{0}, w_{1}, w_{2}, \ldots, w_{L}\right) .
$$

In terms of this functional $F$, the stationary trajectories $w$ are the solutions of the Euler-Poisson equation

$$
\left(\frac{\partial F}{\partial w_{0}}-\frac{d}{d t} \frac{\partial F}{\partial w_{1}}+\frac{d^{2}}{d t^{2}} \frac{\partial F}{\partial w_{2}}-\cdots+(-1)^{L} \frac{d^{L}}{d t^{L}} \frac{\partial F}{\partial w_{L}}\right)\left(w^{(0)}, \ldots, w^{(L)}\right)=0 .
$$


It is a matter of straightforward computation to see that the equations given by (4.1) and (4.2) indeed coincide. Henceforth we will, for a given QDF $Q_{\Phi}$, refer to the differential equation (4.1) as the Euler-Poisson equation associated with $Q_{\Phi}$.

According to the principle of least action, the motions that are possible in a mechanical system can be obtained as the stationary trajectories of the Lagrangian, the difference between the kinetic and potential energy of the system, which is in general represented as a function of displacement and velocity. Accordingly, the corresponding Euler-Poisson equation is a system of second-order differential equations, called the Euler-Lagrange equations associated with the mechanical system. Thus, the possible motions in a mechanical system form a behavior represented by the Euler-Lagrange equations. We now study the converse problem (called the "inverse problem of the calculus of variations"; see [17]): under which conditions does a linear differential behavior $\mathfrak{B}$ (typically described by a system of higher-order linear differential equations) consist of the stationary trajectories with respect to some functional interpretable as a Lagrangian (i.e., a functional that represents the difference between kinetic and potential energy in a suitable sense), and how does one construct such a functional on the basis of the equations describing $\mathfrak{B}$ ? It turns out that under mild assumptions, this is the case if and only if $\mathfrak{B}$ is a Hamiltonian system. This leads us to define the notion of generalized position and generalized Lagrangian and to address the issue of the existence of second-order latent variable representations of Hamiltonian behaviors. See also [7] and [6], where the inverse problem is considered for nonlinear i/o systems, and a characterization of Hamiltonian systems is given in terms of the properties of the i/o differential equations describing them.

TheOREM 4.2. Let $\mathfrak{B} \in \mathcal{L}^{\text {w }}$ be autonomous, and assume that $\chi_{\mathfrak{B}}$, the characteristic polynomial of $\mathfrak{B}$, has no root in zero, $\chi_{\mathfrak{B}}(0) \neq 0$. Then the following statements are equivalent:

(1) $\mathfrak{B}$ is Hamiltonian.

(2) $\mathrm{n}(\mathfrak{B})$ is even, and there exists a full column rank matrix $P(\xi) \in \mathbb{R}^{\mathrm{q} \times \mathrm{w}}[\xi]$, and nonsingular matrices $M=M^{T}, K=K^{T} \in \mathbb{R}^{\mathrm{q} \times \mathrm{q}}$, with $\mathrm{q}:=\mathrm{n}(\mathfrak{B}) / 2$, such that $\mathfrak{B}$ is equal to the space of all stationary trajectories with respect to the $Q D F$

$$
Q_{L}(w)=\left|\frac{d}{d t} P\left(\frac{d}{d t}\right) w\right|_{M}^{2}-\left|P\left(\frac{d}{d t}\right) w\right|_{K}^{2} ;
$$

equivalently, $\mathfrak{B}=\left\{w \in \mathfrak{C}^{\infty}\left(\mathbb{R}, \mathbb{R}^{w}\right) \mid \partial L\left(\frac{d}{d t}\right) w=0\right\}$, with $L(\zeta, \eta)$ defined by

$$
L(\zeta, \eta):=P(\zeta)^{T}(\zeta \eta M-K) P(\eta) .
$$

Furthermore, if any of these conditions holds, then $P, M$, and $K$ satisfying the conditions in (2). can be chosen in such a way that in addition

$$
\frac{d}{d t} Q_{H}(w)=0 \text { for all } w \in \mathfrak{B},
$$

where $Q_{H}(w):=\left|\frac{d}{d t} P\left(\frac{d}{d t}\right) w\right|_{M}^{2}+\left|P\left(\frac{d}{d t}\right) w\right|_{K}^{2}$.

Proof. $((1) \Rightarrow(2))$ Since $\mathfrak{B}$ is Hamiltonian and $\chi_{\mathfrak{B}}(0) \neq 0, \mathfrak{B}$ has only even invariant polynomials. We reduce to the scalar case by use of the Smith form. Consider a minimal representation of the behavior $\mathfrak{B}$ as $\mathfrak{B}=\operatorname{ker} R\left(\frac{d}{d t}\right)$, and let $R=U \Delta V$ be the Smith decomposition of $R$, with $\Delta$ being a diagonal matrix. Define the behavior $\mathfrak{B}^{\prime}:=V\left(\frac{d}{d t}\right) \mathfrak{B}$ with manifest variable $w^{\prime}$, and observe that $\mathfrak{B}^{\prime}=\operatorname{ker} \Delta\left(\frac{d}{d t}\right)$. We now examine each of the behaviors $\mathfrak{B}_{i}^{\prime}:=\operatorname{ker} \lambda_{i}\left(\frac{d}{d t}\right), i=1, \ldots$, w, one at a time. 
Let $\lambda_{i}(\xi):=\lambda_{i 0}+\lambda_{i 2} \xi^{2}+\cdots+\lambda_{i, 2 L_{i}} \xi^{2 L_{i}}, \lambda_{i, 2 L_{i}} \neq 0$. Consider the following two matrices:

$$
M_{i}:=\left(\begin{array}{ccccc}
\lambda_{i, 2} & \lambda_{i, 4} & \lambda_{i, 6} & \cdots & \lambda_{i, 2 L_{i}} \\
\lambda_{i, 4} & \lambda_{i, 6} & \lambda_{i, 8} & \cdots & 0 \\
\vdots & \vdots & \vdots & \cdots & \vdots \\
\lambda_{i, 2 L_{i}-2} & \lambda_{i, 2 L_{i}} & 0 & \cdots & 0 \\
\lambda_{i, 2 L_{i}} & 0 & 0 & \cdots & 0
\end{array}\right)
$$

and

$$
K_{i}:=\left(\begin{array}{ccccc}
\lambda_{i, 0} & 0 & 0 & \ldots & 0 \\
0 & -\lambda_{i, 4} & -\lambda_{i, 6} & \ldots & -\lambda_{i, 2 L_{i}} \\
\vdots & \vdots & \vdots & & \vdots \\
0 & -\lambda_{i, 2 L_{i}-2} & -\lambda_{i, 2 L_{i}} & \ldots & 0 \\
0 & -\lambda_{i, 2 L_{i}} & 0 & \cdots & 0
\end{array}\right)
$$

It is immediate to see that $M_{i}$ is nonsingular; the nonsingularity of $K_{i}$ follows from $\lambda_{i, 2 L_{i}} \neq 0$ and the fact that $\lambda_{i, 0} \neq 0$, since $\chi_{\mathfrak{B}}(0) \neq 0$. Let $E_{i}(\xi):=\operatorname{col}\left(1, \xi^{2}, \ldots, \xi^{2 L_{i}-2}\right)$ and $e_{i}:=\operatorname{col}(1,0, \ldots, 0) \in \mathbb{R}^{L_{i} \times 1}$. Then the following equation holds:

$$
\left(M_{i} \xi^{2}+K_{i}\right) E_{i}(\xi)=\lambda_{i}(\xi) e_{i} .
$$

This implies $E_{i}^{T}(-\xi)\left(M_{i} \xi^{2}+K_{i}\right) E_{i}(\xi)=\lambda_{i}(\xi)$. Define $E(\xi):=\operatorname{block} \operatorname{diag}\left(E_{i}(\xi)\right)$, $M=$ block $\operatorname{diag}\left(M_{i}\right)$, and $K=$ block $\operatorname{diag}\left(K_{i}\right)$; then $\Delta(\xi)=E^{T}(-\xi)\left(\left(M \xi^{2}+K\right) E(\xi)\right.$. Finally, let $P(\xi):=E(\xi) V(\xi)$. Since $\mathfrak{B}^{\prime}=\operatorname{ker} \Delta\left(\frac{d}{d t}\right)$, also $\mathfrak{B}=\operatorname{ker} P^{T}\left(-\frac{d}{d t}\right)\left(M \frac{d^{2}}{d t^{2}}+\right.$ $K) P\left(\frac{d}{d t}\right)$. By defining $\left.L(\zeta, \eta):=P^{T}(\zeta) M \zeta \eta-K\right) P(\eta)$ we then obtain $\mathfrak{B}=\operatorname{ker} \partial L\left(\frac{d}{d t}\right)$; equivalently, $\mathfrak{B}$ is the space of stationary trajectories of the QDF $Q_{L}(w)$.

$((2) \Rightarrow(1))$ Since $\mathfrak{B}=\operatorname{ker} \partial \Phi\left(\frac{d}{d t}\right)$, the claim is proved if we show that the invariant polynomials of $\partial L$ are all even. Observe first that $\partial L$ is para-Hermitian. Now let $1 \leq \mathrm{k} \leq \mathrm{w}$, and consider the set $M_{\mathrm{k}}$ consisting of all $\mathrm{k} \times \mathrm{k}$ minors of $\partial L(\xi)$. Observe that since $\partial L=(\partial L)^{\sim}$, if $m \in M_{\mathrm{k}}$, then also $m^{\sim} \in M_{\mathrm{k}}$. Now use an argument analogous to that used in the proof of Proposition 2.3 in order to conclude that the invariant polynomials of $\partial L$ are either even or odd. Conclude from $\chi_{\mathfrak{B}}(0) \neq 0$ that there are no odd invariant polynomials; then it follows from statement (2) of Theorem 3.4 that $\mathfrak{B}$ is Hamiltonian.

The rest of the claim of the theorem follows easily from the definition of $L$. This concludes the proof.

If, in statement (2) of Theorem 4.2, we interpret $q=P\left(\frac{d}{d t}\right) w$ as generalized position, then $\frac{d}{d t} q=\frac{d}{d t} P\left(\frac{d}{d t}\right) w$ is generalized velocity, and consequently $\left|\frac{d}{d t} P\left(\frac{d}{d t}\right) w\right|_{M}^{2}=$ $\left|\frac{d}{d t} q\right|_{M}^{2}$ and $\left|P\left(\frac{d}{d t}\right) w\right|_{K}^{2}=|q|_{K}^{2}$ can be interpreted, respectively, as kinetic and potential energy. From this point of view, the QDF $Q_{L}(w)$ can be interpreted as a Lagrangian, and the QDF $Q_{H}(w)$ can be interpreted as a Hamiltonian of the system.

The equation $\partial L\left(\frac{d}{d t}\right) w=0$ is the Euler-Poisson equation associated with the QDF $Q_{L}$. Motivated by the fact that $Q_{L}(w)$ can be interpreted as a Lagrangian of the system $\mathfrak{B}$, we also call it an Euler-Lagrange equation associated with the system $\mathfrak{B}$.

The next theorem relates Hamiltonianity with the existence of a latent variable representation of second order in the latent variable $q=P\left(\frac{d}{d t}\right) w$, with $P$ as in Theorem 4.2 . 
Theorem 4.3. Let $\mathfrak{B} \in \mathcal{L}^{\text {W }}$ be autonomous. Assume that $\chi_{\mathfrak{B}}(0) \neq 0$, i.e., its characteristic polynomial has no root in 0 . Then the following statements are equivalent:

(1) $\mathfrak{B}$ is Hamiltonian.

(2) $\mathrm{n}(\mathfrak{B})$ is even, and there exist $M=M^{T}, K=K^{T} \in \mathbb{R}^{\mathrm{q} \times \mathrm{q}}$ nonsingular, $C_{1} \in$ $\mathbb{R}^{\mathrm{w} \times \mathrm{q}}$ and $C_{2} \in \mathbb{R}^{\mathrm{w} \times \mathrm{q}}$, with $\mathrm{q}:=\mathrm{n}(\mathfrak{B}) / 2$, such that

$$
\begin{aligned}
& M \frac{d^{2}}{d t^{2}} q+K q=0, \\
& C_{1} q+C_{2} \frac{d}{d t} q=w
\end{aligned}
$$

is an observable latent variable representation of $\mathfrak{B}$ with latent variable $q$. Furthermore, if $M \frac{d^{2}}{d t^{2}} q+K q=0, w=C_{1} q+C_{2} \frac{d}{d t} q$ is an observable latent variable representation of $\mathfrak{B}$ with latent variable $q$, then the equations $\bar{M} \frac{d^{2}}{d t^{2}} \bar{q}+\bar{K} \bar{q}=0$, $w=\bar{C}_{1} \bar{q}+\bar{C}_{2} \frac{d}{d t} \bar{q}$ form an observable latent variable representation of $\mathfrak{B}$ with latent variable $\bar{q}$ if and only if there exist $S, T \in \mathbb{R}^{\mathrm{q} \times \mathrm{q}}$ such that the matrix

$$
\left(\begin{array}{cc}
S & T \\
-T M^{-1} K & S
\end{array}\right)
$$

is nonsingular, and the relations $S M^{-1} K=\bar{M}^{-1} \bar{K} S, T M^{-1} K=\bar{M}^{-1} \bar{K} T, C_{1}=$ $\bar{C}_{1} S-\bar{C}_{2} T M^{-1} K$, and $C_{2}=\bar{C}_{1} T+\bar{C}_{2} S$ hold.

Proof. ((1) $\Rightarrow(2))$ Define $M_{i}$ and $K_{i}$ by (4.5) and (4.6), respectively. From (4.7) it follows that $w_{i}^{\prime} \in \mathfrak{B}_{i}^{\prime}=\operatorname{ker} \lambda_{i}\left(\frac{d}{d t}\right)$ if and only if $\left(M_{i} \frac{d^{2}}{d t^{2}}+K_{i}\right) E_{i}\left(\frac{d}{d t}\right) w_{i}^{\prime}=0$. Using this, it is easily seen that the equations $M_{i} \frac{d^{2} q}{d t^{2}}+K_{i} q=0, q_{i}=E_{i}\left(\frac{d}{d t}\right) w_{i}^{\prime}$ form an observable latent variable representation of $\mathfrak{B}_{i}^{\prime}$ with latent variable $q_{i}$. Next consider the equations $M_{i} \frac{d^{2} q_{i}}{d t^{2}}+K_{i} q_{i}=0, e_{i}^{T} q_{i}=w_{i}^{\prime}$, where as before $e_{i}:=\operatorname{col}(1,0, \ldots, 0) \in$ $\mathbb{R}^{L_{i} \times 1}$. Using the special structure of $M_{i}$ and $K_{i}$, it can be verified that also these equations form an observable latent variable representation of $\mathfrak{B}_{i}^{\prime}$. Now define $C:=$ block $\operatorname{diag}\left(e_{i}^{T}\right)$. Then clearly $M \frac{d^{2} q}{d t^{2}}+K q=0, C q=w^{\prime}$ is an observable latent variable representation of $\mathfrak{B}^{\prime}$. An observable latent variable representation of the original system $\mathfrak{B}$ is then obtained by replacing the equation $w^{\prime}=C q$ by $w=V^{-1}\left(\frac{d}{d t}\right) C q$. Note that $V^{-1}(\xi) C$ is a polynomial matrix and that any derivative $q^{(i)}$ with $i \geq 2$ can be expressed in terms of $q$ or $q^{(1)}$ using the first equation in (4.8). From this we conclude that matrices $C_{1}$ and $C_{2}$ exist such that $M \frac{d^{2} q}{d t^{2}}+K q=0, w=C_{1} q+C_{2} \frac{d}{d t} q$ is an observable latent variable representation of $\mathfrak{B}$.

$((2) \Rightarrow(1))$ By defining $x_{1}=q, x_{2}=\frac{d q}{d t}$, and $C=\left(\begin{array}{ll}C_{1} & C_{2}\end{array}\right)$, the state-space representation of $\mathfrak{B}$ given by $\frac{d}{d t} x_{1}=x_{2}, \frac{d}{d t} x_{2}=M^{-1} K x_{1}, w=C x$ is obtained. Clearly, $\left(x_{1}, x_{2}\right)$ is observable from $w$, so this state-space representation is minimal. Observe also that

$$
\left(\begin{array}{cc}
0 & I \\
-M^{-1} K & 0
\end{array}\right)^{T}\left(\begin{array}{cc}
0 & M \\
-M & 0
\end{array}\right)+\left(\begin{array}{cc}
0 & M \\
-M & 0
\end{array}\right)\left(\begin{array}{cc}
0 & I \\
-M^{-1} K & 0
\end{array}\right)=0 .
$$

Since $M$ is nonsingular, we conclude from statement (3) of Theorem 3.4 that $\mathfrak{B}$ is Hamiltonian.

Assume now that $M \frac{d^{2}}{d t^{2}} q+K q=0, w=C_{1} q+C_{2} \frac{d}{d t} q$ is an observable latent variable representation of $\mathfrak{B}$ with latent variable $q$. Suppose the equations $\bar{M} \frac{d^{2}}{d t^{2}} \bar{q}+$ 
$\bar{K} \bar{q}=0, w=\bar{C}_{1} \bar{q}+\bar{C}_{2} \frac{d}{d t} \bar{q}$ form an observable latent variable representation of $\mathfrak{B}$ with latent variable $\bar{q}$. Then clearly $\frac{d}{d t} x=A x, w=C x$ and $\frac{d}{d t} \bar{x}=\bar{A} \bar{x}, w=\bar{C} \bar{x}$ with

$$
\begin{gathered}
A:=\left(\begin{array}{cc}
0 & I \\
-M^{-1} K & 0
\end{array}\right), \quad \bar{A}:=\left(\begin{array}{cc}
0 & I \\
\bar{M}^{-1} \bar{K} & 0
\end{array}\right), \\
C:=\left(\begin{array}{ll}
C_{1} & C_{2}
\end{array}\right), \bar{C}:=\left(\begin{array}{ll}
\bar{C}_{1} & \bar{C}_{2}
\end{array}\right)
\end{gathered}
$$

are two minimal state representations of $\mathfrak{B}$. Consequently there exists a nonsingular $F \in \mathbb{R}^{2 \mathrm{q} \times 2 \mathrm{q}}$ such that $\bar{A} F=F A$ and $\bar{C} F=C$. Using the special structure of the state-space representations it is easily seen that $F$ must be of the form (4.9) and that the relations in the claim must hold. Conversely, by defining $F$ by (4.9), we see that $\bar{A} F=F A$ and $\bar{C} F=C$, so that the corresponding state realizations have the same manifest behavior $\mathfrak{B}$.

Remark 4.4. Note that in the proof of implication $(2) \Rightarrow(1)$ the assumption that $\chi_{\mathfrak{B}}(\xi)$ has no root in $\xi=0$ is not used. Our proof of implication $(1) \Rightarrow(2)$ does use this assumption. At present we do not have a proof of or a counterexample to this implication without the assumption $\chi_{\mathfrak{B}}(0) \neq 0$.

In classical mechanics, the variable $q$ consists of the position of the masses, and (4.8) is obtained by writing down Newton's second law. Theorem 4.3 shows that a Hamiltonian behavior can always be interpreted in some sense as a "mechanical system," with $q$ "generalized position," $\frac{d}{d t} q$ "generalized velocity," $M$ a "mass matrix," and $K$ a matrix of "elastic constants." Further, the theorem characterizes all such representations. Of course $\frac{d}{d t} q^{T} M \frac{d}{d t} q$ can be interpreted as kinetic energy, $q^{T} K q$ as potential energy, $\left(\frac{d}{d t} q\right)^{T} M\left(\frac{d}{d t} q\right)-q^{T} K q$ as a Lagrangian, and $\left(\frac{d}{d t} q\right)^{T} M\left(\frac{d}{d t} q\right)+q^{T} K q$ (which is constant along solutions $q$ of the first equation in (4.8)) as total energy.

The similarity between (4.8) and the second-order representation typical of conservative mechanical systems should not, however, be pushed too far. Indeed, the reader can verify that the matrix $M$ defined in the proof of Theorem 4.3 is in general not positive-definite, as a bona fide mass matrix should be. Nor would the "kinetic" and "potential" terms $\left|P\left(\frac{d}{d t}\right) w\right|_{M}^{2}$ and $\left|P\left(\frac{d}{d t}\right) w\right|_{K}^{2}$ have in general the physical dimensions of energies. For further discussion on these issues see the following example.

Example 4.5. Consider the configuration of Example 3.3. As shown in that example, the behavior $\mathfrak{B}$ of the position $w$ of the first mass is represented by

$$
r\left(\frac{d}{d t}\right) w=m_{1} m_{2} \frac{d^{4}}{d t^{4}} w+\left(k_{1} m_{1}+k_{2} m_{1}+k_{1} m_{2}\right) \frac{d^{2}}{d t^{2}} w+k_{1} k_{2} w=0 .
$$

In order to obtain an observable second-order latent variable representation (4.8) of $\mathfrak{B}$ we proceed as in the proof of Theorem 4.3 and define the latent variable $q=$ $\operatorname{col}\left(w, \frac{d^{2}}{d t^{2}} w\right)$. Then

$$
M:=\left(\begin{array}{ll}
r_{2} & r_{4} \\
r_{4} & 0
\end{array}\right), \quad K:=\left(\begin{array}{ll}
r_{0} & 0 \\
0 & -r_{4}
\end{array}\right), \quad C_{1}=1, \quad C_{2}=0 .
$$

The matrix $M$ is not positive-definite, as can be verified choosing $k_{1}=k_{2}=1 \frac{\mathrm{N}}{\mathrm{m}}$ and $m_{1}=m_{2}=1 \mathrm{~kg}$. Observe also that with such a choice of $M$, a physical interpretation of $\frac{d}{d t} q^{T} M \frac{d}{d t} q$ is impossible, since the physical dimensions of such a quantity are not those of an energy. However, by choosing

$$
\bar{K}:=\left(\begin{array}{cc}
k_{2} & \frac{k_{2} m_{1}}{k_{1}} \\
\frac{k_{2} m_{1}}{k_{1}} & \frac{m_{1}^{2}\left(k_{1}+k_{2}\right)}{k_{1}^{2}}
\end{array}\right), \quad \bar{M}:=\left(\begin{array}{cc}
m_{1}+m_{2} & \frac{m_{1} m_{2}}{k_{1}} \\
\frac{m_{1} m_{2}}{k_{1}} & \frac{m_{1}^{2} m_{2}}{k_{1}^{2}}
\end{array}\right), \quad \bar{C}_{1}:=1, \quad \bar{C}_{2}:=0,
$$


we obtain an alternative second-order latent variable representation of $\mathfrak{B}$, with the same latent variable as before. Note that $\bar{M}^{-1} \bar{K}=M^{-1} K$ so that this alternative representation is obtained from the original one by taking in Theorem $4.3 S=I, T=0$. For this second choice of representation, the generalized kinetic energy $\left(\frac{d}{d t} q\right)^{T} M\left(\frac{d}{d t} q\right)$ and generalized potential energy $q^{T} K q$ coincide with the physical kinetic energy and potential energy of the system with the two masses, that is, $E_{\text {kin }}\left(w_{1}, w_{2}\right)=$ $\frac{1}{2}\left(m_{1}\left(\frac{d}{d t} w_{1}\right)^{2}+m_{2}\left(\frac{d}{d t} w_{2}\right)^{2}\right)$ and $E_{\text {pot }}\left(w_{1}, w_{2}\right)=\frac{1}{2}\left(k_{1} w_{1}^{2}-2 k_{1} w_{1} w_{2}+\left(k_{1}+k_{2}\right) w_{2}^{2}\right)$. This can be verified observing that the generalized position $q=\left(w, \frac{d^{2}}{d t^{2}} w\right)$ is related to the actual position $\left(w_{1}, w_{2}\right)$ as $w_{1}=w, w_{2}=w+\frac{m_{1}}{k_{1}} \frac{d^{2}}{d t^{2}} w$.

It is a matter for further investigation to see whether and how a physically consistent choice of the matrices $M$ and $K$ can always be performed. Such an issue is particularly pressing when considering the use of the procedures presented in this paper for computer-assisted modeling and simulation.

5. Internal forces and controllable Hamiltonian systems. In this section we define internal forces as auxiliary variables and we show how they can be obtained from a higher-order Lagrangian such as that introduced in Theorem 4.2. The notion of internal force obtained in this way brings us in a natural way to the definition of a controllable Hamiltonian system; in this section we also give various characterizations of such systems in terms of their kernel, image, or state-space representations.

According to Theorem 4.2, an autonomous system $\mathfrak{B} \in \mathcal{L}^{\text {w }}$ with $\chi_{\mathfrak{B}}(0) \neq 0$ is Hamiltonian if and only if there exists a polynomial matrix $P \in \mathbb{R}^{\mathrm{q} \times \mathrm{w}}[\xi]$ with full column rank, $M=M^{T}, K=K^{T} \in \mathbb{R}^{\mathrm{q} \times \mathrm{q}}$ nonsingular, with $\mathrm{q}:=\mathrm{n}(\mathfrak{B}) / 2$, such that $\mathfrak{B}$ has a kernel representation $P^{T}\left(-\frac{d}{d t}\right)\left(M \frac{d^{2}}{d t^{2}}+K\right) P\left(\frac{d}{d t}\right) w=0$. Obviously, a latent variable representation with latent variable $f$ of $\mathfrak{B}$ is then given by the equations

$$
\begin{aligned}
P^{T}\left(-\frac{d}{d t}\right) M \frac{d^{2}}{d t^{2}} P\left(\frac{d}{d t}\right) w & =f, \\
f & =-P^{T}\left(-\frac{d}{d t}\right) K P\left(\frac{d}{d t}\right) w .
\end{aligned}
$$

For a given $w \in \mathfrak{B}$, the associated $f=-P^{T}\left(-\frac{d}{d t}\right) K P\left(\frac{d}{d t}\right) w$ is called the internal force associated with $w$. Observe that there are as many internal forces as there are external variables, and that in the case of systems described by differential equations of order higher than two, the internal force depends on higher-order derivatives of the external variable (see Chapter 2, section 31 of [21], where, in the context of the dynamics of a moving charge in an electromagnetic field, an internal force is considered which depends on a potential function which depends on position and velocity). Such a definition harmonizes with the classical mechanics point of view of seeing the internal force as an auxiliary variable of the same dimension as the external variables and coming from some potential function depending on the configuration (external) variables. Indeed, when applied to the prototypical mechanical system $M \frac{d^{2}}{d t^{2}} q+K q=0, w=q$, equations (5.1) result in the internal force being defined as $f=-K q$ and coming from the potential $V(q)=q^{T} K q$.

The notion of internal force sheds light on the structure of autonomous Hamiltonian systems. Define $\mathfrak{B}_{1}, \mathfrak{B}_{2} \in \mathcal{L}^{\mathrm{w}+\mathrm{f}}$ by

$$
\mathfrak{B}_{1}:=\left\{\left(\begin{array}{c}
w \\
f
\end{array}\right) \mid\left(P^{T}\left(-\frac{d}{d t}\right) M \frac{d^{2}}{d t^{2}} P\left(\frac{d}{d t}\right)-I\right)\left(\begin{array}{c}
w \\
f
\end{array}\right)=0\right\}
$$




$$
\mathfrak{B}_{2}:=\left\{\left(\begin{array}{c}
w \\
f
\end{array}\right) \mid\left(P^{T}\left(-\frac{d}{d t}\right) K P\left(\frac{d}{d t}\right) \quad I\right)\left(\begin{array}{c}
w \\
f
\end{array}\right)=0\right\} .
$$

Note that both $\mathfrak{B}_{1}$ and $\mathfrak{B}_{2}$ are controllable linear differential systems. The set of trajectories $(w, f)$ compatible with the laws of both $\mathfrak{B}_{1}$ and $\mathfrak{B}_{2}$ is the behavior $\mathfrak{B}_{1} \cap \mathfrak{B}_{2}$; we call it the full interconnection of $\mathfrak{B}_{1}$ and $\mathfrak{B}_{2}$. Now assume that $P$, $M$, and $K$ in (5.1) have been computed as in the proof of $((1) \Rightarrow(2))$ of Theorem 4.2. It is a matter of straightforward verification to see that in such a case $P^{T}(-\xi) M P(\xi)$ is nonsingular; this implies that in $\mathfrak{B}_{1}, f$ is input and $w$ is output; it can also be verified that in $\mathfrak{B}_{2}, w$ is input and $f$ is output. In such a case the interconnection of $\mathfrak{B}_{1}$ and $\mathfrak{B}_{2}$ is called a feedback interconnection (see [23]).

We conclude that any autonomous Hamiltonian behavior $\mathfrak{B}$ is the feedback interconnection of two systems, the first one $\left(\mathfrak{B}_{1}\right)$ having a free $f$ variable and the second one $\left(\mathfrak{B}_{2}\right)$ imposing on such a variable the additional constraint represented by the second equation in (5.1). From this standpoint, in $\mathfrak{B}_{1}, f$ is an external force which can be chosen freely, while $\mathfrak{B}_{2}$ constrains it to be a function of the external variables $w$. This point of view has much in common with the notion of "Hamiltonian interconnection" introduced in [18], where the concept of an open Hamiltonian system is introduced from a system-theoretic point of view.

Example 5.1. We consider again the system described in Examples 3.3 and 4.5. Following the procedure illustrated above, $\mathfrak{B}_{1}$ and $\mathfrak{B}_{2}$ defined in (5.2) and (5.3) are described, respectively, by

$$
\begin{aligned}
& \left(m_{1}+m_{2}\right) w+2 \frac{m_{1} m_{2}}{k_{1}} \frac{d^{2}}{d t^{2}} w+\frac{m_{1}^{2} m_{2}}{k_{1}^{2}} \frac{d^{4}}{d t^{4}} w=f, \\
& f=k_{2} w+\frac{2 k_{2} m_{1}}{k_{1}} \frac{d^{2}}{d t^{2}} w+\left(\frac{m_{1}^{2}}{k_{1}}+\frac{k_{2} m_{1}^{2}}{k_{1}^{2}}\right) \frac{d^{4}}{d t^{4}} w .
\end{aligned}
$$

Using the fact that $w$ satisfies the fourth-order differential equation

$$
m_{1} m_{2} \frac{d^{4}}{d t^{4}} w+\left(k_{1} m_{1}+k_{2} m_{1}+k_{1} m_{2}\right) \frac{d^{2}}{d t^{2}} w+k_{1} k_{2} w=0,
$$

the expression for $f$ obtained from the second equation in (5.4) can be rewritten in terms of the generalized position $q=\operatorname{col}\left(w, \frac{d^{2}}{d t^{2}} w\right)$ as

$$
f=\left(k_{2}-\frac{k_{2} m_{1}}{m_{2}}-\frac{k_{2}^{2} m_{1}}{k_{1} m_{2}}\right) w+\left(-m_{1}+\frac{k_{2} m_{1}}{k_{1}}-\frac{m_{1}^{2}}{m_{2}}-2 \frac{k_{2} m_{1}^{2}}{k_{1} m_{2}}-\frac{k_{2}^{2} m_{1}^{2}}{k_{1}^{2} m_{2}}\right) \frac{d^{2}}{d t^{2}} w .
$$

Such an expression can also be given in terms of the positions of the two masses described in Example 3.3 as

$$
f=\left(k_{2}+\frac{k_{1} m_{1}}{m_{2}}+\frac{k_{2} m_{1}}{m_{2}}\right) w_{1}+\left(k_{2}-k_{1}-\frac{k_{1} m_{1}}{m_{2}}-\frac{2 k_{2} m_{1}}{m_{2}}-\frac{k_{2}^{2} m_{1}}{k_{1} m_{2}}\right) w_{2} .
$$

The physical interpretation of such a quantity is not easy, though it should be remarked that the physical dimensions of such a latent variable are indeed those of a force.

When modeling physical phenomena, closed (i.e., autonomous) systems are the exception rather than the rule: the environment in which the system is embedded almost always interacts with it, exerting some influence. Sometimes it is reasonable to assume that the way in which the environment interacts with the system - in other 
words, the generating mechanism of the external influences - depends only on the attributes of the system itself, the paramount example of such a situation being the motion of a point mass or charge in a force field depending on its position. By modeling the external influence as a function of the system "configuration" we obtain an autonomous system, whose evolution depends only on its laws of motion and the "initial state."

If we take such a point of view when considering the description of $\mathfrak{B}$ as the interconnection of $\mathfrak{B}_{1}$ and $\mathfrak{B}_{2}$ defined in (5.2) and (5.3), it is natural to consider $\mathfrak{B}_{1}$ as a model of an open system with external influences modeled by $f$ and $\mathfrak{B}_{2}$ as a description of the way in which $f$ depends on the variables $w$. In principle different constraints could be imposed by $\mathfrak{B}_{2}$ on $f$, and therefore it is natural to consider the open system $\mathfrak{B}_{1}$ as the starting point for a study of controllable Hamiltonian systems and to investigate the consequences of the Hamiltonianity of $\mathfrak{B}$ on $\mathfrak{B}_{1}$. This leads us to the definition of controllable Hamiltonian behavior, which we presently give.

Observe first that $\mathfrak{B}_{1}$ defined by (5.2) has as many outputs (external variables $w$ ) as inputs (the auxiliary variables $f$ ), in accordance with the point of view adopted in classical mechanics of considering the configuration variables as manifest ones and the external forces as inputs, each acting on a configuration variable (as in the case of collocated sensors and actuators; see section 12.1 of [12]). Now consider two compactsupport trajectories $\left(w_{i}, f_{i}\right) \in \mathfrak{B}_{1}, i=1,2$, and compute the integral $\int_{-\infty}^{+\infty} w_{1} f_{2}-$ $w_{2} f_{1} \mathrm{~d} t$. Integrating by parts using (5.2) and the fact that the trajectories $\left(w_{i}, f_{i}\right)$ are compact support, it is not difficult to verify that such an integral is zero. Such an observation brings us to the notion of a controllable Hamiltonian system.

Definition 5.2. Let $\mathfrak{B} \in \mathcal{L}^{\mathrm{w}}$ be controllable, with w even. Denote

$$
J_{\mathrm{W}}:=\left(\begin{array}{cc}
0 & I_{\frac{\mathrm{w}}{2}} \\
-I_{\frac{\mathrm{w}}{2}} & 0
\end{array}\right) \text {. }
$$

$\mathfrak{B}$ is called Hamiltonian if for all trajectories $w_{1}, w_{2} \in \mathfrak{B} \cap \mathfrak{D}\left(\mathbb{R}, \mathbb{R}^{\mathrm{w}}\right)$ we have

$$
\int_{-\infty}^{+\infty} L_{J_{w}}\left(w_{1}, w_{2}\right) d t=0
$$

We discuss the relationship of Definition 5.2 with other notions of Hamiltonianity in Remarks 5.5 and 5.6 below. We proceed by illustrating Definition 5.2 with an example and then give a number of characterizations of Hamiltonianity for controllable systems in Theorem 5.4, the main result of this section.

Example 5.3. Take the same system considered in Example 4.5, but with an external force applied to the first mass. Choose as external variables the position $q$ of the first mass and the external force $f$; then it is easy to see that the behavior of the system is represented by the equation

$$
m_{1} m_{2} \frac{d^{4} q}{d t^{4}}+\left(m_{1} k_{1}+m_{1} k_{2}+m_{2} k_{1}\right) \frac{d^{2} q}{d t^{2}}+k_{1} k_{2} q=m_{2} \frac{d^{2} f}{d t^{2}}+\left(k_{1}+k_{2}\right) f .
$$

In order for this system to be controllable, the polynomials $d(\xi):=m_{1} m_{2} \xi^{4}+\left(m_{1} k_{1}+\right.$ $\left.m_{1} k_{2}+m_{2} k_{1}\right) \xi^{2}+k_{1} k_{2}$ and $n(\xi):=m_{2} \xi^{2}+k_{1}+k_{2}$ must be coprime. In that case the system also admits an observable image representation induced by the polynomial matrix $M(\xi):=\operatorname{col}(n(\xi), d(\xi))$. We now show that this system is Hamiltonian. Observe that for any pair of compact-support trajectories $w_{i}=M\left(\frac{d}{d t}\right) \ell_{i}, i=1,2$, it holds that $L J_{2}\left(w_{1}, w_{2}\right)=L_{\Phi}\left(\ell_{1}, \ell_{2}\right)$, where $\Phi(\zeta, \eta):=M(\zeta)^{T} J_{2} M(\eta)$. In order 
to prove that $\int_{-\infty}^{+\infty} L J_{2}\left(w_{1}, w_{2}\right) d t=0$, observe that $\Phi(-\xi, \xi)=0$. Conclude from Theorem 3.1 of $[24]$ that there exists $\Psi \in \mathbb{R}[\zeta, \eta]$ such that $\Phi(\zeta, \eta)=(\zeta+\eta) \Psi(\zeta, \eta)$, equivalently, $\frac{d}{d t} L_{\Psi}=L_{\Phi}$. Now using the fact that the latent variable trajectories $\ell_{i}$ also have compact support, we can infer that $\int_{-\infty}^{+\infty} L_{\Phi}\left(\ell_{1}, \ell_{2}\right) d t=\left.L_{\Psi}\left(\ell_{1}, \ell_{2}\right)\right|_{-\infty} ^{+\infty}=0$.

In order to state the main result of this section, consisting of several alternative characterizations of Hamiltonianity for controllable behaviors, we need to introduce the notion of an orthogonal of a controllable behavior. Given a controllable linear differential behavior $\mathfrak{B} \in \mathcal{L}^{\text {w}}$, we define its orthogonal complement $\mathfrak{B}^{\perp}$ as

$$
\mathfrak{B}^{\perp}:=\left\{w \in \mathfrak{C}^{\infty}\left(\mathbb{R}, \mathbb{R}^{\mathrm{w}}\right) \mid \int_{-\infty}^{+\infty} w^{T} w^{\prime} \mathrm{d} t=0 \text { for all } w^{\prime} \in \mathfrak{B} \cap \mathfrak{D}\left(\mathbb{R}, \mathbb{R}^{w}\right)\right\} .
$$

The orthogonal $\mathfrak{B}^{\perp}$ is again an element of $\mathfrak{L}^{w}$, and it is controllable (see section 10 of [24]).

TheOREM 5.4. Let $\mathfrak{B} \in \mathcal{L}^{\mathrm{w}}$ be controllable, with w even. Let $J_{\mathrm{w}}$ be given by (5.5). Then the following statements are equivalent:

(1) $\mathfrak{B}$ is Hamiltonian.

(2) $\mathfrak{B}=\left(J_{\text {w }} \mathfrak{B}\right)^{\perp}$.

(3) $M^{\sim} J_{\mathrm{w}} M=0$ for each $M$ such that $w=M\left(\frac{d}{d t}\right) \ell$ is an image representation of $\mathfrak{B}$.

(4) $R J_{\mathfrak{w}} R^{\sim}=0$ for each $R$ such that $R\left(\frac{d}{d t}\right) w=0$ is a kernel representation of $\mathfrak{B}$.

(5) For every i/o partition $\operatorname{col}(u, y)=\Pi w$ of $\mathfrak{B}$ the transfer function $G$ from $u$ to y satisfies $G^{\sim} \Sigma=\Sigma G$, with $\Sigma$ the $\frac{\mathrm{w}}{2} \times \frac{\mathrm{w}}{2}$ signature matrix determined by

$$
\Pi J_{\mathrm{w}} \Pi^{T}=\left(\begin{array}{cc}
0 & \Sigma \\
-\Sigma & 0
\end{array}\right) .
$$

(6) $\mathrm{n}:=\mathrm{n}(\mathfrak{B})$ is even, and there exists a minimal $i / s / o$ representation

$$
\frac{d}{d t} x=A x+B u, \quad y=C x+D u, \quad \operatorname{col}(u, y)=\Pi w
$$

of $\mathfrak{B}$, such that $J_{\mathrm{n}} A+A^{T} J_{\mathrm{n}}=0, \Sigma D=D^{T} \Sigma$, and $B^{T} J_{\mathrm{n}}=-\Sigma C$, with $\Sigma$ the $\frac{\mathrm{W}}{2} \times \frac{\mathrm{W}}{2}$ signature matrix determined by (5.6).

(7) $\mathrm{n}:=\mathrm{n}(\mathfrak{B})$ is even, and for every minimal i/s/o representation

$$
\frac{d}{d t} x=A x+B u, \quad y=C x+D u, \quad \operatorname{col}(u, y)=\Pi w
$$

of $\mathfrak{B}$, there exists a nonsingular skew-symmetric matrix $K \in \mathbb{R}^{\mathrm{n} \times \mathrm{n}}$ such that $K A+A^{T} K=0, \Sigma D=D^{T} \Sigma$, and $B^{T} K=-\Sigma C$, with $\Sigma$ the $\frac{\mathrm{w}}{2} \times \frac{\mathrm{w}}{2}$ signature matrix determined by (5.6).

Proof. $((1) \Leftrightarrow(3))$ Let $w=M\left(\frac{d}{d t}\right) \ell$ be an image representation of $\mathfrak{B}$. Observe that $\int_{-\infty}^{\infty} L_{J_{\mathrm{w}}}\left(w_{1}, w_{2}\right) d t=0$ for all $w_{1}, w_{2} \in \mathfrak{B} \cap \mathfrak{D}\left(\mathbb{R}, \mathbb{R}^{\mathrm{w}}\right)$ if and only if $\int_{-\infty}^{\infty} L_{\Psi}\left(\ell_{1}, \ell_{2}\right) d t=$ 0 for all $\ell_{1}, \ell_{2}$ of compact support, where $\Psi(\zeta, \eta):=M(\zeta)^{T} J_{w} M(\eta)$. By Theorem 3.1 of [24] this holds if and only if $M^{\sim} J_{\mathrm{w}} M=0$.

$((3) \Leftrightarrow(5))$ Let $\Pi$ be a w $\times$ w permutation matrix such that for $\operatorname{col}(u, y) \in \Pi \mathfrak{B}$, $u$ is input and $y$ is output. Let $w=M\left(\frac{d}{d t}\right) \ell$ be any image representation of $\mathfrak{B}$ with $M$ full column rank. Then correspondingly $\Pi M=\operatorname{col}(U, Y)$, with $\operatorname{det}(U) \neq 0$. The transfer matrix from $u$ to $y$ is equal to the matrix of rational functions $G=Y U^{-1}$. We have

$$
M^{\sim} J_{\mathfrak{w}} M=M^{\sim} \Pi^{T} \Pi J_{\mathfrak{w}} \Pi^{T} \Pi M=M^{\sim} \Pi^{T}\left(\begin{array}{cc}
0 & \Sigma \\
-\Sigma & 0
\end{array}\right) \Pi M=U^{\sim} \Sigma Y-Y^{\sim} \Sigma U,
$$


with $\Sigma$ a nonsingular $\frac{\mathrm{W}}{2} \times \frac{\mathrm{W}}{2}$ signature matrix. Since $M^{\sim} J_{\mathrm{w}} M=0$ we obtain $U^{\sim} \Sigma Y-$ $Y^{\sim} \Sigma U=0$, equivalently, $G^{\sim} \Sigma=\Sigma G$. Conversely, if $G^{\sim} \Sigma=\Sigma G$ then take a coprime factorization $G=Y U^{-1}$. Then $M:=\Pi^{T} \operatorname{col}(U, Y)$ yields an observable image representation $w=M\left(\frac{d}{d t}\right) \ell$ of $\mathfrak{B}$ which clearly satisfies $M^{\sim} J_{\mathfrak{w}} M=0$. It then follows easily that $M^{\sim} J_{\mathrm{w}} M=0$ for any $M$ such that $w=M\left(\frac{d}{d t}\right) \ell$ is an image representation of $\mathfrak{B}$.

$((3) \Rightarrow(2))$ Observe that $M^{T}\left(-\frac{d}{d t}\right) w^{\prime}=0$ is a kernel representation of $\mathfrak{B}^{\perp}$ and $M^{T}\left(-\frac{d}{d t}\right) J_{\mathrm{w}} w^{\prime \prime}=0$ is a kernel representation of $\left(J_{\mathrm{w}} \mathfrak{B}\right)^{\perp}$. From $M^{\sim} J_{\mathrm{W}} M=0$ it thus follows that $\mathfrak{B} \subseteq\left(J_{\mathfrak{w}} \mathfrak{B}\right)^{\perp}$. The equivalence $((3) \Leftrightarrow(5))$ shows that every transfer matrix of $\mathfrak{B}$ is square, and consequently $\mathrm{m}(\mathfrak{B})=\mathrm{p}(\mathfrak{B})$. Hence we have $\mathrm{m}\left(\left(\mathrm{J}_{\mathrm{w}} \mathfrak{B}\right)^{\perp}\right)=\mathrm{p}\left(\mathrm{J}_{\mathrm{w}} \mathfrak{B}\right)=\mathrm{p}(\mathfrak{B})=\mathrm{m}(\mathfrak{B})$. Using the calculus of behavioral equations (see $[15])$ it is not difficult to prove that two controllable behaviors $\mathfrak{B}_{1}$ and $\mathfrak{B}_{2}$, with the same number of inputs such that $\mathfrak{B}_{1} \subseteq \mathfrak{B}_{2}$, must be equal. This implies that, in fact, the equality $\mathfrak{B}=\left(J_{\mathrm{w}} \mathfrak{B}\right)^{\perp}$ holds.

$((2) \Rightarrow(1)) \mathfrak{B} \subseteq\left(J_{\mathfrak{w}} \mathfrak{B}\right)^{\perp}$ by definition implies that $\int_{-\infty}^{\infty} L_{J_{w}}\left(w_{1}, w_{2}\right) d t=0$ for all $w_{1}, w_{2} \in \mathfrak{B} \cap \mathfrak{D}\left(\mathbb{R}, \mathbb{R}^{\mathrm{w}}\right)$.

$((2) \Leftrightarrow(4))$ Let $R\left(\frac{d}{d t}\right) w=0$ be a kernel representation of $\mathfrak{B}$. Then $w^{\prime}=R^{T}\left(-\frac{d}{d t}\right) \ell$ is an image representation of $\mathfrak{B}^{\perp}$ and $w^{\prime \prime}=J_{\mathrm{w}} R^{T}\left(-\frac{d}{d t}\right) \ell$ is an image representation of $\left(J_{\mathrm{w}} \mathfrak{B}\right)^{\perp}$ (see section 10 of $\left.[24]\right)$. Clearly $\left(J_{\mathrm{w}} \mathfrak{B}\right)^{\perp} \subseteq \mathfrak{B}$ implies $R J_{\mathrm{w}} R^{\sim}=0$. Conversely, if $R J_{\mathrm{w}} R^{\sim}=0$ then $\left(J_{\mathrm{w}} \mathfrak{B}\right)^{\perp} \subseteq \mathfrak{B}$. Also, it is easily seen that $R J_{\mathrm{w}} R^{\sim}=0$ implies condition (5), so that $\mathrm{p}(\mathfrak{B})=\mathrm{m}(\mathfrak{B})$. By the same argument used in the proof of the implication $(3) \Rightarrow(2)$ this yields that $\mathfrak{B}=\left(J_{\mathfrak{w}} \mathfrak{B}\right)^{\perp}$.

$((5) \Rightarrow(7))$ Let $(A, B, C, D)$ be the quadruple of matrices associated with a minimal i/s/o representation of $\mathfrak{B}$. This yields an i/o partition with transfer matrix $G(\xi)=D+C(\xi I-A)^{-1} B$. By minimality of the i/s/o representation it follows that the pair $(C, A)$ is observable; moreover, by controllability of $\mathfrak{B}$, it follows that the pair $(A, B)$ is controllable. Recall that there exists a nonsingular signature matrix $\Sigma$ such that $\Sigma G=G^{\sim} \Sigma$. This implies that $(A, B, \Sigma C, \Sigma D)$ and $\left(-A^{T}, C^{T} \Sigma,-B^{T}, D^{T} \Sigma\right)$ are minimal realizations of the same transfer matrix. Consequently there exists a unique nonsingular matrix $K$ such that $-A^{T}=K A K^{-1}, C^{T} \Sigma=K B,-B^{T}=\Sigma C K^{-1}$, $\Sigma D=D^{T} \Sigma$. It is easily verified that also $-A^{T}=\left(-K^{T}\right) A\left(-K^{-T}\right), C^{T} \Sigma=\left(-K^{T}\right) B$, $-B^{T}=\Sigma C\left(-K^{-T}\right)$. Due to the uniqueness of $K$, it follows that $K^{T}=-K$, i.e., $K$ is skew-symmetric.

$((7) \Rightarrow(6))$ Let $(A, B, C, D)$ be the quadruple of matrices associated with a minimal i/s/o representation of $\mathfrak{B}$. Let $K$ be nonsingular and skew-symmetric and let $\Sigma$ be a nonsingular signature matrix such that $K A+A^{T} K=0, \Sigma D=D^{T} \Sigma$, and $B^{T} K=-\Sigma C$. There exists a nonsingular matrix $S$ such that $S^{T} J_{\mathrm{n}} S=K$. Define $\hat{A}:=S A S^{-1}, \hat{B}=S B, \hat{C}=C S^{-1}$, and $\hat{D}=D$. This quadruple also defines a minimal i/s/o representation of $\mathfrak{B}$. Moreover, it is easily verified that $J_{\mathrm{n}} \hat{A}+\hat{A}^{T} J_{\mathrm{n}}=0$, $\Sigma \hat{D}=\hat{D}^{T} \Sigma$, and $\hat{B}^{T} J_{\mathrm{n}}=-\Sigma \hat{C}$.

$((6) \Rightarrow(3))$ If an $\mathrm{i} / \mathrm{s} / \mathrm{o}$ representation of $\mathfrak{B}$ exists satisfying the conditions in (6), then it follows that the transfer matrix from $u$ to $y$ satisfies $G^{\sim} \Sigma=\Sigma G$. Take a coprime factorization $G=Y U^{-1}$. Then $M:=\Pi^{T} \operatorname{col}(U, Y)$ yields an observable image representation $w=M\left(\frac{d}{d t}\right) \ell$ of $\mathfrak{B}$ which clearly satisfies $M^{\sim} J_{\mathrm{w}} M=0$. It then follows easily that $M^{\sim} J_{\mathrm{w}} M=0$ for any $M$ such that $w=M\left(\frac{d}{d t}\right) \ell$ is an image representation of $\mathfrak{B}$.

Remark 5.5. The definition of a nonlinear Hamiltonian i/o system put forward in $[7,6]$ is based on the self-adjointness of the i/o map of the system. We now discuss how such a point of view relates with that given in Definition 5.2 and elaborated on 
in Theorem 5.4. Assume that the variables $w$ of the controllable system described in kernel form by $R\left(\frac{d}{d t}\right) w=0, R$ of full row rank, are partitioned as $w=\operatorname{col}(u, y)$, with $u$ consisting of $\frac{w}{2}$ input variables, and $y$ consisting of $\frac{w}{2}$ output variables. Then the matrix $R$ can be partitioned as $R=\left(\begin{array}{ll}P & -Q\end{array}\right)$, with $P$ square and invertible.

Under these assumptions, the condition $R J_{\mathrm{w}} R^{\sim}=0$ appearing in statement (4) of Theorem 5.4 reads $Q P^{\sim}-P Q^{\sim}=0$, which is equivalent with formula (51) of [6], once it is recalled that an image representation of the adjoint system (equivalently, of the orthogonal behavior) to ker $R\left(\frac{d}{d t}\right)$ is given by $\operatorname{Im} R^{T}\left(-\frac{d}{d t}\right)$.

Remark 5.6. The characterization of Hamiltonian transfer functions given in statement (5) of Theorem 5.4 is the same given in [3] in the context of i/s/o systems and in [8] in the polynomial model context. See also [20], where an external characterization of the adjoint of a linear system is given, and several of the techniques used (see, in particular, section III loc. cit.) foreshadow those based on the calculus of Q/BDFs used in the present paper.

Remark 5.7. It follows from Theorem 5.4 (for example, by applying condition (4)) that the systems $\mathfrak{B}_{1}$ and $\mathfrak{B}_{2}$ represented by, respectively, (5.2) and (5.3) are controllable Hamiltonian systems. In other words, if $\mathfrak{B}$ is an autonomous Hamiltonian system with $\chi_{\mathfrak{B}}(0) \neq 0$, then there exist two controllable Hamiltonian behaviors $\mathfrak{B}_{i}$, $i=1,2$, such that $\mathfrak{B}$ is the feedback interconnection of $\mathfrak{B}_{1}$ and $\mathfrak{B}_{2}$.

We conclude this section with two examples of controllable Hamiltonian systems.

Example 5.8. Newton's second law defines a controllable Hamiltonian system $\mathfrak{B}=\left\{(F, q) \mid F=m \frac{d^{2}}{d t^{2}} q\right\}$, as it is easy to verify using, for example, statement (4) of Theorem 5.4.

Example 5.9. Consider a parallel interconnection of a capacitor $C$ with an inductance $L$ subject to an external current $I_{e}$. Assume that we choose as external variables for such a system the external current and the magnetic flux $\phi_{L}$ in the inductance; it is easy to verify that in such a case the system equation is $\left(\frac{d^{2}}{d t^{2}}+\frac{1}{C L}\right) \phi_{L}-\frac{1}{C} I_{e}=0$. We show that this system is Hamiltonian. An observable image representation of the system is induced by the matrix $M(\xi)=\operatorname{col}\left(1, C \xi^{2}+\frac{1}{L}\right)$. Consider that $M(\zeta)^{T} J_{2} M(\eta)=(\zeta+\eta) \Psi(\zeta, \eta)$ with $\Psi(\zeta, \eta):=C(\zeta-\eta)$. Consequently such a BDF satisfies $\frac{d}{d t} L_{\Psi}=L_{J_{2}}$ on $\mathfrak{B}$, and therefore $\int_{-\infty}^{+\infty} L_{J_{2}}\left(w_{1}, w_{2}\right)=0$ for all $w_{1}, w_{2} \in \mathfrak{B}$.

6. Conclusions. In this paper we have used the formalism of bilinear and quadratic differential forms in order to study Hamiltonian systems. The approach followed in this paper is representation-free, i.e., independent of the existence of a special representation of the system, such as a transfer function or a state-space representation. However, we have also given a characterization of Hamiltonianity for various system representations such as kernel, state-space, and transfer function, in the case of autonomous systems (Theorem 3.4) and of controllable ones (Theorem 5.4). We have also proposed a definition of generalized total energy and generalized Lagrangian (see section 4), and we introduced the notion of generalized internal forces for systems described by higher-order differential equations (see section 5).

The major limitation of the present work is its treatment of Hamiltonianity for the controllable and autonomous case only, leaving out the general case of a system comprising a nonzero controllable part and a nonzero "autonomous part" (for the difficulty in defining uniquely such a subbehavior, see [15, p. 192]). The development of a general theory of linear Hamiltonian behaviors that includes completely controllable and completely autonomous behaviors as special cases is a pressing issue in our research. 
In view of the encouraging results of the application of quadratic differential forms in the context of infinite-dimensional systems (see $[13,14]$ ), it can be hoped that some of the results presented in this paper can be generalized also to systems described by linear constant-coefficient partial differential equations; such an area of research is presently under investigation. Another direction in which the research presented in this paper is being extended is that of the connections between Hamiltonian systems and optimal control.

Acknowledgments. The authors thank Prof. Dr. Ir. Jan C. Willems and Prof. Dr. A. J. van der Schaft, on whom several preliminary versions of this paper were inflicted; they stimulated several discussions conducive to the elaboration of very valuable ideas and pointed out important references in the literature. Many thanks also to Dr. H. K. Pillai for his help in understanding the more technical points of [5].

\section{REFERENCES}

[1] V. I. Arnold, Mathematical Methods of Classical Mechanics, Springer-Verlag, Berlin, 1978.

[2] R. W. Brockett, Path integrals, Lyapunov functions, and quadratic minimization, in Proceedings of the 4th Allerton Conference on Circuit and System Theory, University of Illinois, Monticello, IL, 1966, pp. 685-698.

[3] R. W. BRockett And A. RAhimi, Lie algebras and linear differential equations, in Ordinary Differential Equations, L. Weiss, ed., Academic Press, New York, 1972, pp. 379-386.

[4] A. Ciampi, Classification of Hamiltonian linear systems, Indiana Univ. Math. J., 23 (1973), pp. 513-526.

[5] T. Cotroneo And H. K. Pillai, Linear variational behaviors, in Proceedings of the 38th IEEE Conference on Decision and Control, Phoenix, AZ, 1999.

[6] P. E. Crouch, F. Lamnabhi-Lagarrigue, and A. J. v.d. Schaft, Adjoint and Hamiltonian input-output differential equations, IEEE Trans. Automat. Control, 40 (1995), pp. 603-615.

[7] P. E. Crouch And A. J. van Der Schaft, Variational and Hamiltonian Control Systems, Lecture Notes in Control and Inform. Sci. 101, Springer-Verlag, New York, 1987.

[8] P. A. Fuhrmann, On Hamiltonian rational transfer functions, Linear Algebra Appl., 63 (1984), pp. 1-93.

[9] F. R. Gantmacher, The Theory of Matrices, Chelsea, New York, 1959.

[10] T. Kailath, Linear Systems, Prentice-Hall, Englewood Cliffs, NJ, 1980.

[11] R. E. Kalman, Algebraic characterization of polynomials whose zeros lie in certain algebraic domains, Proc. Natl. Acad. Sci. USA, 64 (1969), pp. 818-823.

[12] H. NiJmeijer and A. J. van der Schaft, Nonlinear Dynamical Control Systems, SpringerVerlag, New York, 1990.

[13] H. K. Pillai and S. Shankar, A behavioral approach to control of distributed systems, SIAM J. Control Optim., 37 (1998), pp. 388-408.

[14] H. K. Pillai and J. C. Willems, Lossless and dissipative distributed systems, SIAM J. Control Optim., 40 (2002), pp. 1406-1430.

[15] J. W. Polderman and J. C. Willems, Introduction to Mathematical System Theory: A Behavioral Approach, Springer-Verlag, Berlin, 1997.

[16] P. Rapisarda And J. C. Willems, State maps for linear systems, SIAM J. Control Optim., 35 (1997), pp. 1053-1091.

[17] R. M. Santilli, Foundations of Theoretical Mechanics I, Springer-Verlag, New York, 1978.

[18] A. J. VAN DER SCHAFT, Hamiltonian dynamics with external forces and observations, Math. Systems Theory, 15 (1982), pp. 145-168.

[19] A. J. VAn Der Schaft, System Theoretic Description of Physical Systems, CWI Tract 3, CWI, Amsterdam, 1984.

[20] A. J. VAN DER SCHAFT, Duality for linear systems: External and state space characterization of the adjoint system, in Analysis of Controlled Dynamical Systems, B. Bonnard, B. Bride, J.- P. Gauthier, and I. Kupka, eds., Birkhäuser, Boston, 1991, pp. 393-403.

[21] E. T. Whittaker, A Treatise on the Analytical Dynamics of Particles and Rigid Bodies, with an Introduction to the Problem of Three Bodies, Cambridge University Press, Cambridge, UK, 1988. 
[22] J. C. Willems and J. C. Fuhrmann, Stability theory for high-order systems, Linear Algebra Appl., 167 (1992), pp. 131-149.

[23] J. C. Willems, On interconnections, control, and feedback, IEEE Trans. Automat. Control, 42 (1997), pp. 326-339.

[24] J. C. Willems and H. L. Trentelman, On quadratic differential forms, SIAM J. Control Optim., 36 (1998), pp. 1703-1749. 